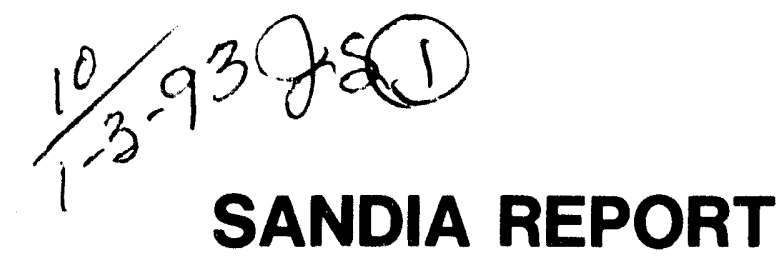

SAND93-1425 • UC-706

Unlimited Release

Printed November 1993

$\checkmark$

i)

\title{
A Non-Contacting Vertical Alignment System for Mass Properties Measuring Instruments
}

George H. James III, Jose E. Suazo, Robert C. Varga

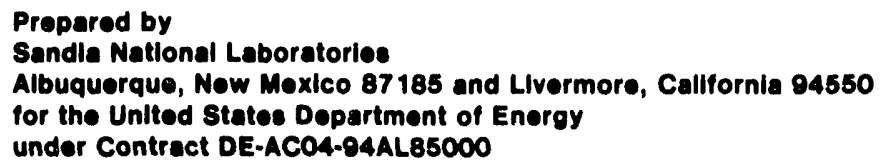


Issued by Sandia National Laboratories, operated for the United States Department of Energy by Sandia Corporation.

NOTICE: This report was prepared as an account of work sponsored by an agency of the United States Government. Neither the United States Government nor any agency thereof, nor any of their employees, nor any of their contractors, subcontractors, or their employees, makes any warranty, express or implied, or assumes any legal liability or responsibility for the accuracy, completeness, or usefulness of any information, apparatus, product, or process disclosed, or represents that its use would not infringe privately owned rights. Reference herein to any specific commercial product, process, or service by trade name, trademark, manufacturer, or otherwise, does not necessarily constitute or imply its endorsement, recommendation, or favoring by the United States Government, any agency thereof or any of their contractors or subcontractors. The views and opinions expressed herein do not necessarily state or reflect those of the United States Government, any agency thereof or any of their contractors.

Printed in the United States of America. This report has been reproduced directly from the best available copy.

Available to DOE and DOE contractors from

Office of Scientific and Technical Information

PO Box 62

Oak Ridge, TN 37831

Prices available from (615) 576-8401, FTS 626-8401

Available to the public from

National Technical Information Service

US Department of Commerce

5285 Port Royal Rd

Springfield, VA 22161

NTIS price codes

Printed copy: A05

Microfiche copy: A01 
SAND93-1425

Unlimited Release

Distribution

Printed November 1993

Category UC-706

\title{
A NON-CONTACTING VERTICAL ALIGNMENT SYSTEM FOR MASS PROPERTIES MEASURING INSTRUMENTS
}

\author{
George H. James III \\ Experimental Structural Dynamics Department \\ Jose E. Suazo \\ Aerospace Systems Development Center \\ Robert C. Varga \\ Environmental Test Department \\ Sandia National Laboratories \\ Albuquerque, NM 87185
}

\begin{abstract}
A non-contact system for alignment of objects on mass properties measuring instruments is described. Test parts can be aligned to within the capabilities of the user and the fixture to make the adjustments. The current implementation can align objects to less than .001 inches at two points with final requested adjustments of a few ten-thousands of an inch. The non-contact capability allows the alignment of objects which are too compliant or fragile for traditional contacting measurement methods. Also, this system allows the definition of a reference axis on objects which are not perfectly symmetric. The reference axis is defined at the top of the object by an appropriate marker and defined at the bottom by a best fit circle through the surface at a specified height. A general description of the hardware, procedures, and results are presented for the non-user. Appendices which contain a complete description of the software, usage. and mathematical implementation are provided for the reader who is interested in using or further developing the system.
\end{abstract}




\section{ACKNOWLEDGMENTS}

Performance requirements for certain Target objects that are designed to fly in space demand that the physical properties of Target balance and moments of inertia be set and verified within close tolerances. In support of that goal it was necessary to design and develop a Non-Contacting Vertical Alignment System for Mass Properties Measuring Instruments. The United States Army Space and Strategic Defense Command (USASSDC) in conjunction with the Ballistic Missile Defense Organization (BMDO), formerly the Strategic Defense Initiative Organization (SDIO), provided the requirements for Targets development through the Penaids Development Program (PDP) as well as the direction and funding that made it possible to develop this system. We are grateful to Mrs. Elaine P. Alspach and Mr. Randall W. Carpenter, both of USASSDC CSSD-TE, and to Major Bob Kelsey and Major Nat Thongchua, both of $\mathrm{BMDO} / \mathrm{GST}$.

The development of this capability has truly been a team effort. Johnny Molina provided assistance in acquiring and using the Motion Analysis system and all video equipment. Roger Goode and Sam Espinoza performed much of the early work on the laser displacement sensors and A to D system. Mike Nusser has provided design and shop support. Ken Padilla handled machine installation. Ken Miller specified and performed the computer upgrade. Joe Kubas developed the menu format for the user programs. Ron Rodeman, Vern Gabbard, Tom Baca, and Rod May have all provided managerial support for the work performed within the Development Test Center. Gerry Mitchell, Roger Abbot, Gerry Hochrein, Bob Lohr, Keith Miller, and Jerry McDowell have all provided managerial support from the Aerospace Systems Development Center. The authors greatly appreciate the support of all these individuals. 


\section{CONTENTS}

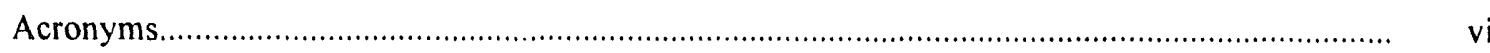

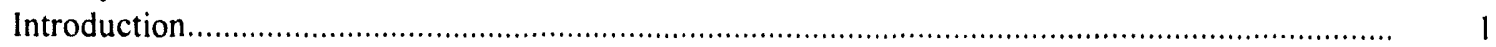

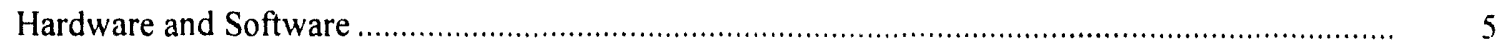

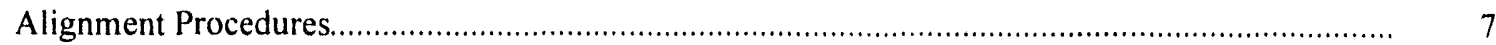

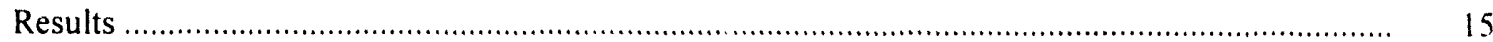

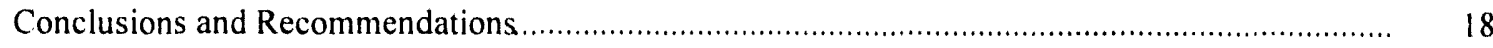

APPENDIX A - Instructions for Mass Properties Measurements and Alignment........................ A-1

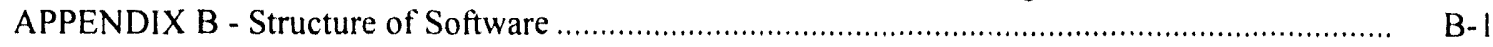

APPENDIX C - Interpretation and Harmonic Curve Fit of Video Data...................................... C-1

APPENDIX D - Interpretation and Harmonic Curve Fit of Laser Data..................................... D-I

APPENDIX E - Alignment Calculation................................................................................ E-1

APPENDIX F - Alignment Calculation Corrected for Conical Objects ..................................... F-1

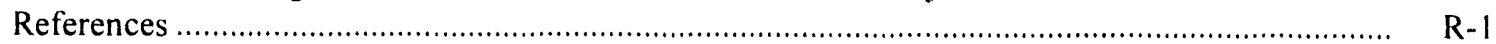

\section{FIGURES}

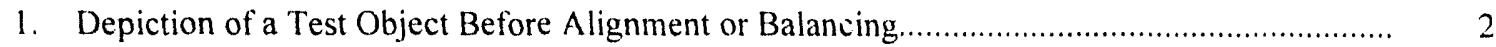

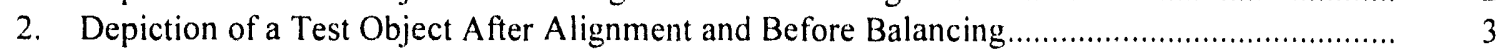

3. Depiction of a Test Object After Alignment and Balancing..................................................

4. View of Test Object, Mass Properties Measuring Instrument, and Alignment System.............. 6

5. Close-up View of Main Equipment Rack.................................................................... 8

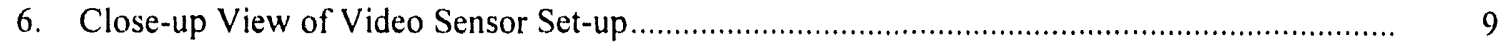

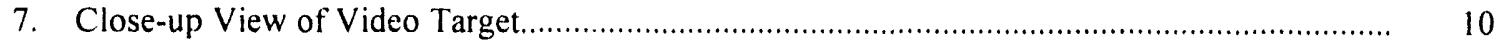

8. Close-up View of Analog Laser Displacement Se: sors ................................................. 11

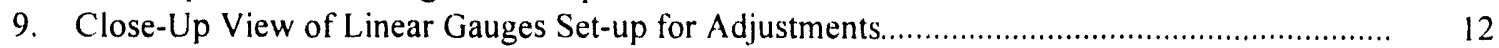

10. Schematic of Alignment Process .............................................................................. 13

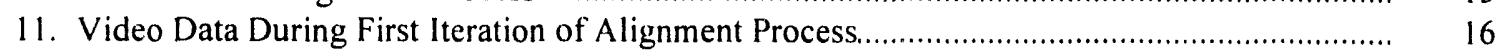

12. Laser Data During First Iteration of Alignment Process ................................................... 17

13. Video Data During Final Iteration of Alignment Process ............................................... 19

14. Laser Data During Final Iteration of Alignment Process.................................................... 20

\section{TABLES}

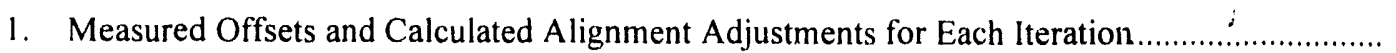




\section{ACRONYMS}

ADS

CG

DOS

EV

LED

MOI

POI

RPM

Analog to Digital Data Acquisition System

Cenier of Gravity

Disk Operating System

Expertvision System

Light Emitting Diode

Moment of Inertia

Product of Inertia

Revolutions Per Minute 


\section{A NON-CONTACTING VERTICAL ALIGNMENT SYSTEM FOR MASS PROPERTIES MEASURING INSTRUMENTS}

\section{INTRODUCTION}

Sandia National Laboratories has recently required a capability for dynamic balancing and measuring the mass properties of large lightweight objects (largest dimension - over 5 feet, weight - less than 10 pounds). Performing these functions on this class of objects has created four specific problems related to the weight, size, compliance, and shape irregularity. The first problem was the increased sensitivity required for this weight class of objects. A second problem was the effects of entrapment and entrainment of air during testing of these large objects. Such effects are negligible on conventional weight objects (weight $>100$ pounds), but become significant for these lightweight objects. Therefore, in December of 1991 two new mass properties measuring instruments were purchased with greater sensitivity as well as the capability to test in a helium environment as well as air [1,2]. This equipment solved the first two problems mentioned above. This report details the hardware, software, and procedures developed to solve the two remaining problems.

The third problem was due to compliance (or inability to support a local surface load) of these lightweight objects. A critical step in the dynamic balancing and measurement of roll moment of inertia was alignment of the object's desired spin axis (test object reference axis) with the spin axis of the mass properties measuring instrument (machine spin axis). Figures 1 contains visual descriptions of the three axes under discussion (machine spin axis, test object reference axis, and test object principal or actual body spin axis). Figure I shows an idealized object placed on the spin balancing machine. The test object's reference axis is not aligned with the machine spin axis. After alignment, the test object reference axis and machine spin axis are coincident as depicted in Figure 2. The balancing process then alters the test object principal axis to be coincident with the other axes and is shown in Figure 3. This alignment is accomplished on conventional hard surface objects using sensors such as dial indicators which contact the object surface, However, the surface of these objects was not rigid enough to reliably support contacting transducers. Therefore, non-contacting techniques for aligning these objects were developed and will be discussed in this report.

The fourth problem was shape irregularity of the object due to the construction techniques necessary to produce this class of test objects. Typically, the reference axis or desired spin axis is defined by the geometry of the object (as in an axis of symmetry) and may be called a geometric axis. However, in the case of these objects, such a geometric axis cannot be defined. Some objects have hard points fore and aft which can be used to define a reference axis. Other objects did not have such hard points and the actual surface must be used to define a reference axis. The most obvious approach is to define the centroid of the area $\left(X_{c}\right.$ and $Y_{c}$ defined with respect to the machine axis) at a given height. Such an approach would begin by defining the shape of the surface in polar coordinates $(r$ and $\theta$ ). The centroid is then defined below [3]:

$$
X_{c}=\frac{\int_{0}^{2 \pi} x \sqrt{\left(\frac{d x}{d \theta}\right)^{2}+\left(\frac{d y}{d \theta}\right)^{2}} d \theta}{\int_{0}^{2 \pi} \sqrt{\left(\frac{d x}{d \theta}\right)^{2}+\left(\frac{d y}{d \theta}\right)^{2}} d \theta}=\frac{\int_{0}^{2 \pi} r \cos (\theta) \sqrt{r^{2}+\left(\frac{d r}{d \theta}\right)^{2}} d \theta}{\int_{0}^{2 \pi} \sqrt{r^{2}+\left(\frac{d r}{d \theta}\right)^{2}} d \theta},
$$




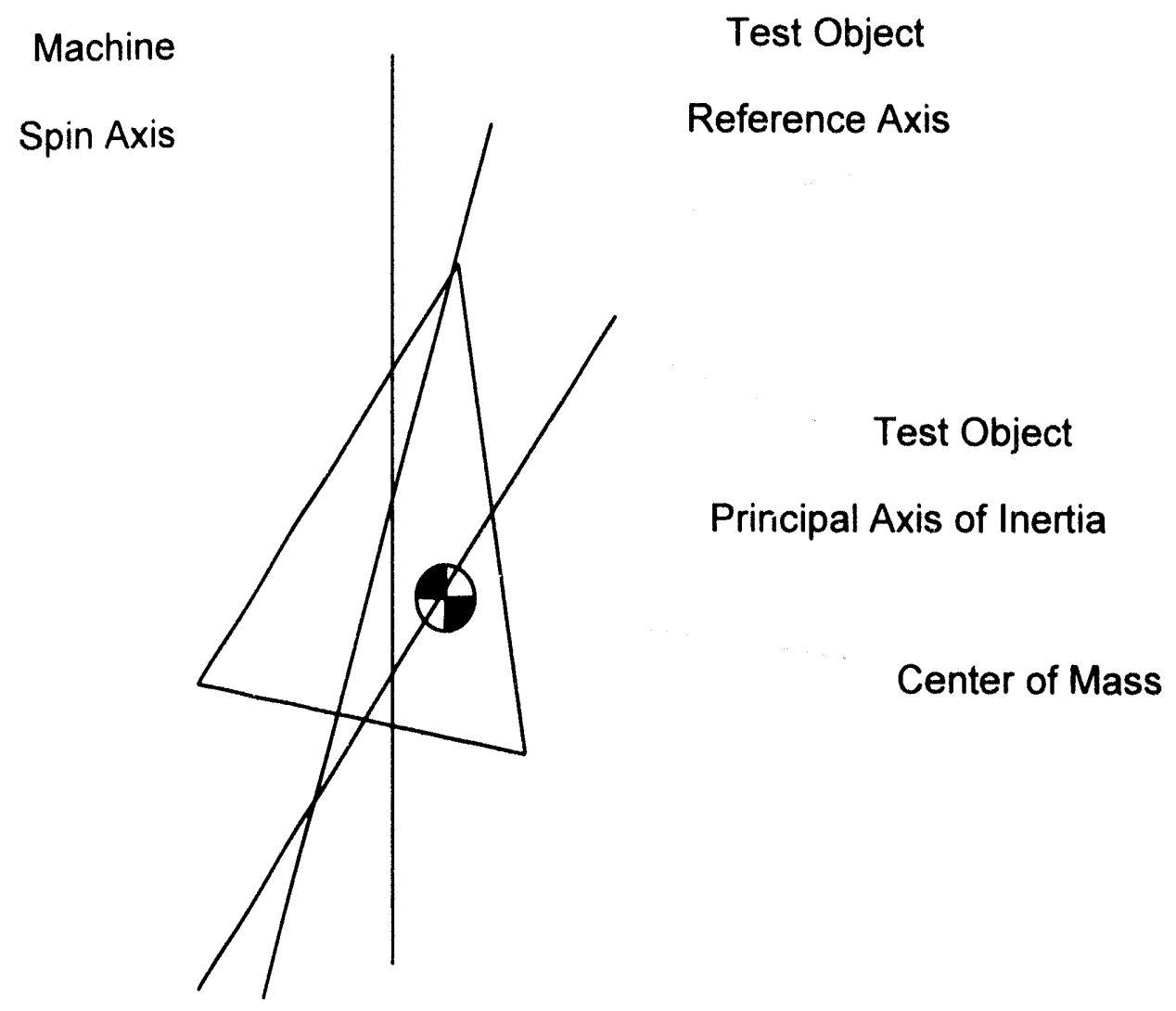

Figure 1. Depiction of an Idealized Test Object Before Alignment or Balancing 
Machine

Spin Axis

Test Object

Reference Axis
Angle of Inclination

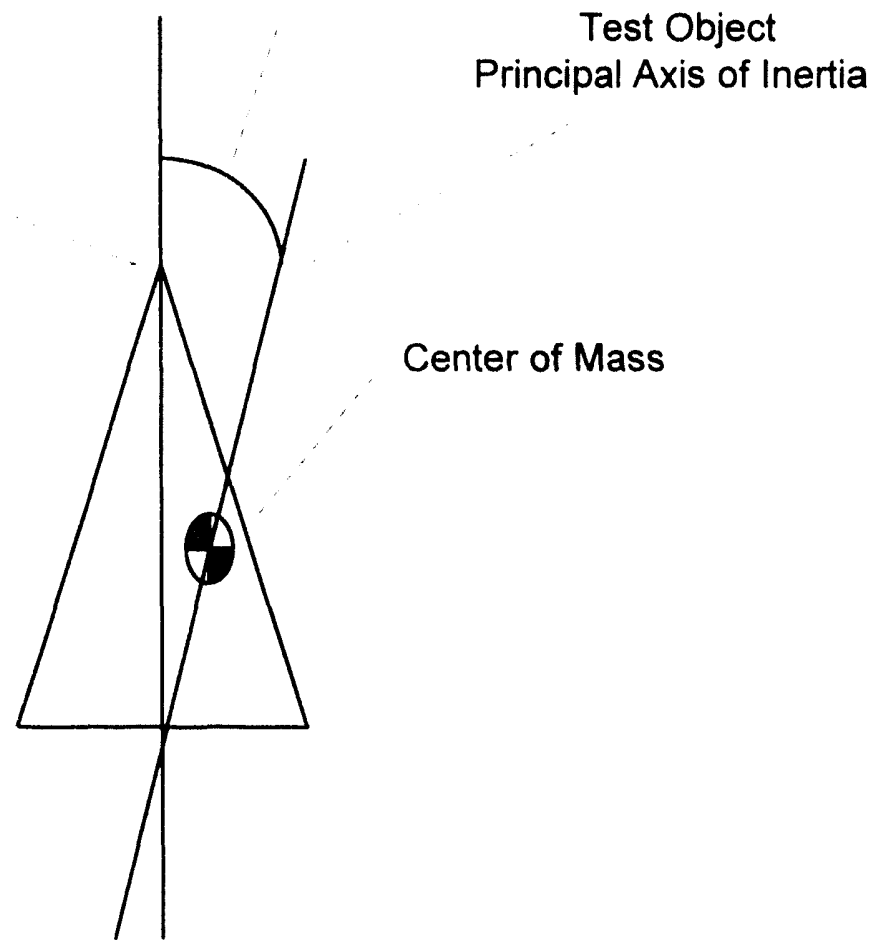

Figure 2. Depiction of an Idealized Test Object After Alignment and Before Balancing 


\section{Angle of Inclination $=0$ Degrees}

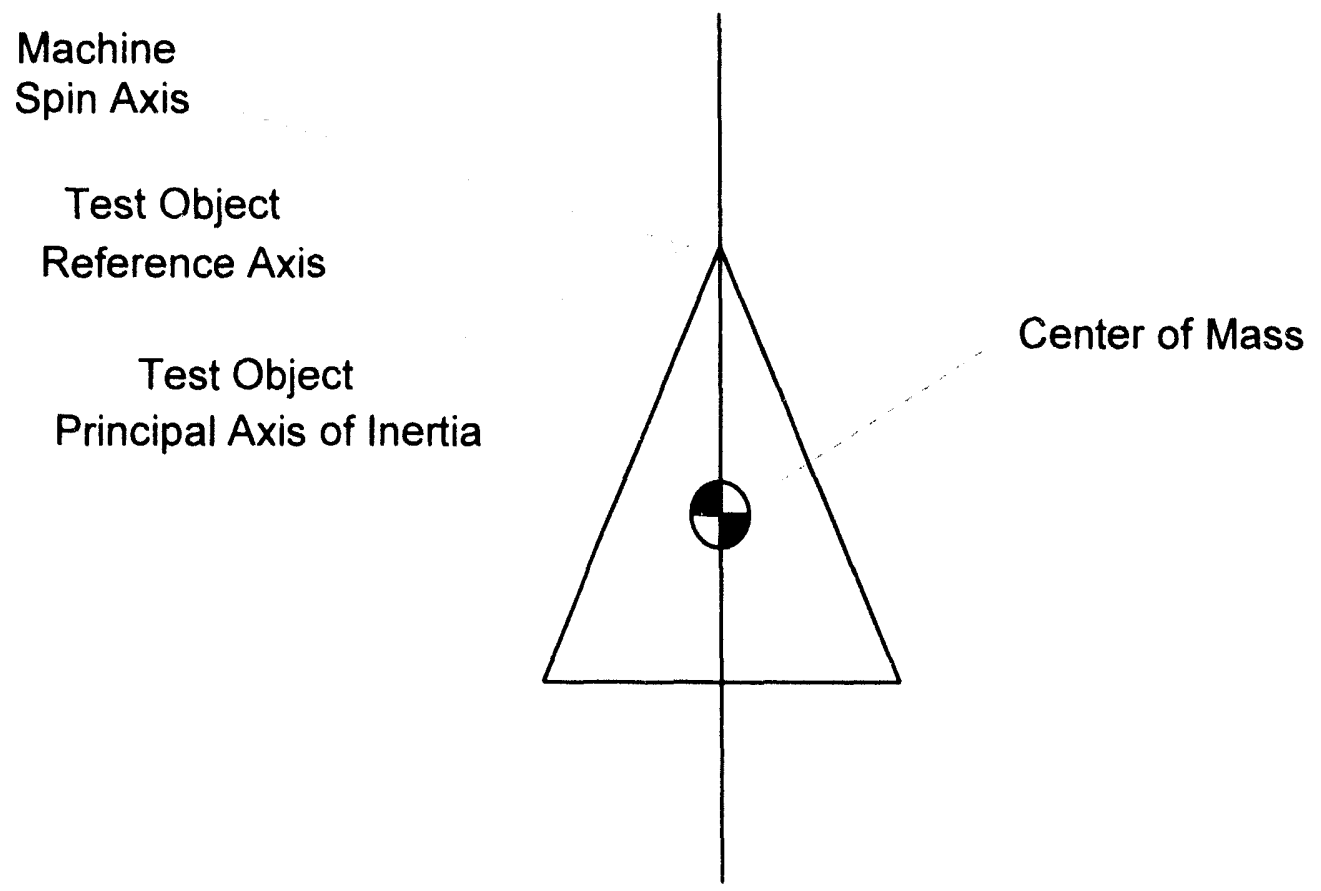

Figure 3. Depiction of an Idealized Test Object After Alignment and Balancing 


$$
Y_{c}=\frac{\int_{0}^{2 \pi} y \sqrt{\left(\frac{d x}{d \theta}\right)^{2}+\left(\frac{d y}{d \theta}\right)^{2}} d \theta}{\int_{0}^{2 \pi} \sqrt{\left(\frac{d x}{d \theta}\right)^{2}+\left(\frac{d y}{d \theta}\right)^{2}} d \theta}=\frac{\int_{0}^{2 \pi} r \sin (\theta) \sqrt{r^{2}+\left(\frac{d r}{d \theta}\right)^{2}} d \theta}{\int_{0}^{2 \pi} \sqrt{r^{2}+\left(\frac{d r}{d \theta}\right)^{2}} d \theta},
$$

However, there are several problems with this approach. First, the radius vector $r$ must be measured absolutely and the available measuring devices only measure a change in the radius vector. Second, the derivative of the radius vector measurements must be taken numerically and this increases the noise in the data. Third, non-linear mathematical manipulations are required to obtain the integrands. And finally, numerical integration is needed to complete the estimation of the centroid location. Therefore, an alternate technique was developed which utilizes a geometric analysis of a perfect citcle, a harmonic curve fit to the data, and small offset approximations to arrive at a simpler procedure. This procedure will be discussed in detail in an appendix.

A description of the hardware and commercial software used to accomplish this work follows the introduction. The actual operations are controlled by several user programs which are called by a main menu. This menu is described as well as the general flow of the procedure. Each menu selection is covered in a separate section with listings of all the applicable user programs. Recommendations for future upgrades are described in the concluding section.

The body of the report is intended to provide a general description of this procedure and its capabilities. Therefore, the readers of the body are not expected to be users of the system. An extensive set of appendices are provided which list the steps to perform in using the system (Appendix A), detail the structure of the software (Appendix B), and provide the mathematical derivations of all important calculations (Appendices C, D, E, and F). The intended audience of these appendices are users of the system, readers interested in understanding the mathematics behind the procedure, and those who desire to upgrade the system.

\section{HARDWARE AND SOFTWARE}

The alignment system is built around a Motion Analysis Corporation 2D Expertvision Flextrak system including a VP1 10 video processor interfaced to a 386 computer and Epson LQ-870 dot matrix printer. Also included in the computer was a 16 channel Analog to Digital data acquisition System (ADS). The video equipment included an NEC TI-23A CCD camera, a Pelco MLZ6DT Motorized Zoom Lens, a Motion Analysis Mac Lite (ring light using red LEDs). and a Sony SLV-686HF VCR which was interfaced to the VP110. 3M Scotchlite Reflective Sheeting and an All American Bushing Co. No. 17700 Toolmaker's Ball were used to produce targets for the video camera. An IBM compatible 486/50 computer with processor heat sink was later substituted for the 386. Two Aromat MQ-LA I-S2-AC100$120 \mathrm{~V}$ Laser Analog Sensors were interfaced to channels one and two of the ADS. Four Mitutoyo LG150E Linear Gauges with Mitutoyo LG-D2 Display Units were used to make the proper adjustments to the Space Electronics Tilt/Translation table which was mounted to the Space Electronics POI-50Z balancing and product of inertia measuring instrument. The software packages used included the Motion Analysis Corporation Expertvision software for video processing [4], the Motion Analysis Corporation ADA-16 software to drive the ADS [5], and the Mathworks, Inc. Matlab package for numerical calculations [6]. Microsoft's DOS 5.0 operating system was used to automate some of the upperlevel functions with batch files.

A view of the entire alignment system, the POI-50Z balancer, and a typical test object are shown in Figure 4. Also shown in Figure 4 are the computer and console to control the POI-50Z and a Video 


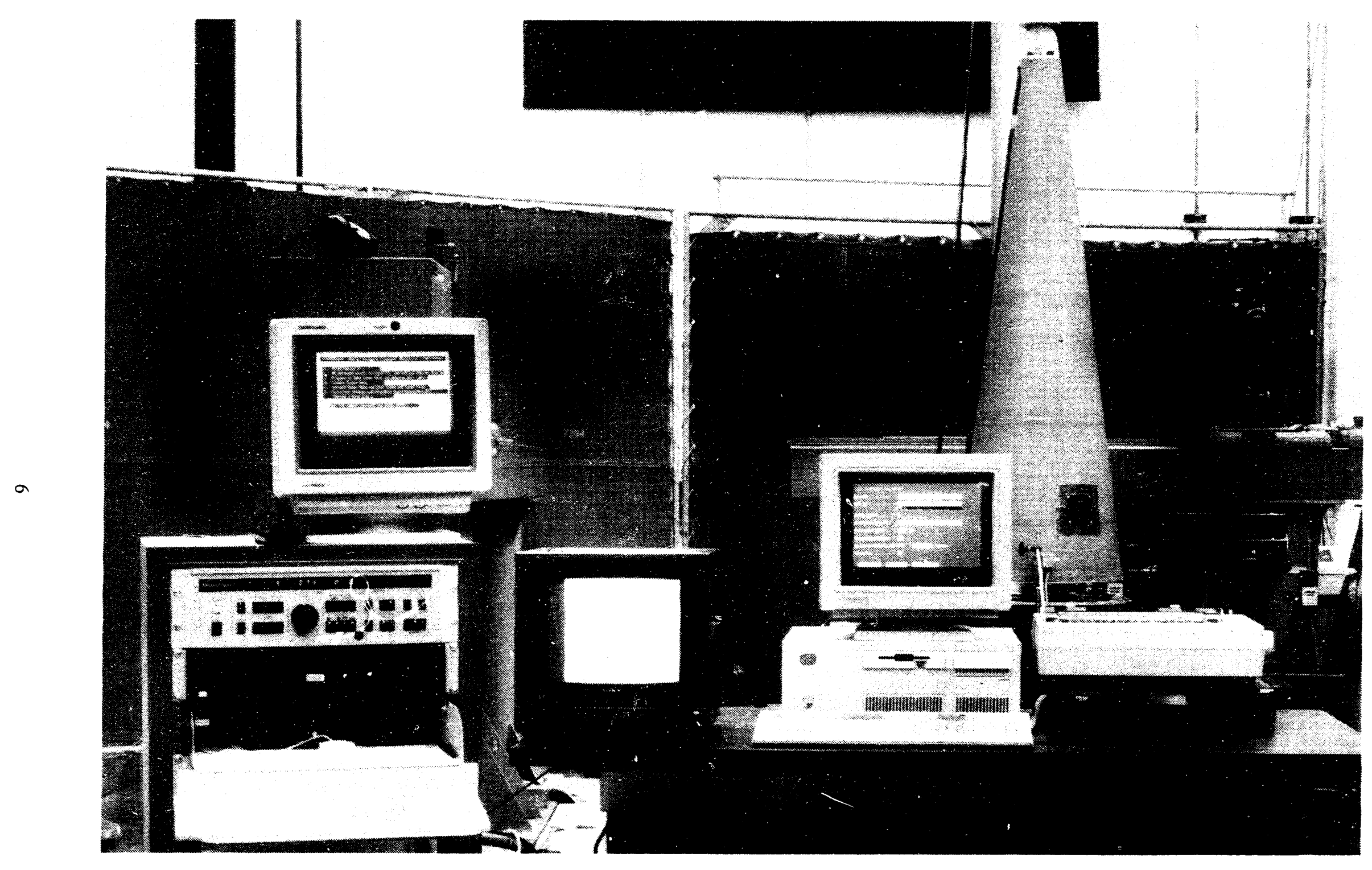

Figure 4. View of Test Object, Mass Properties Measuring Instrument, and Alignment System 


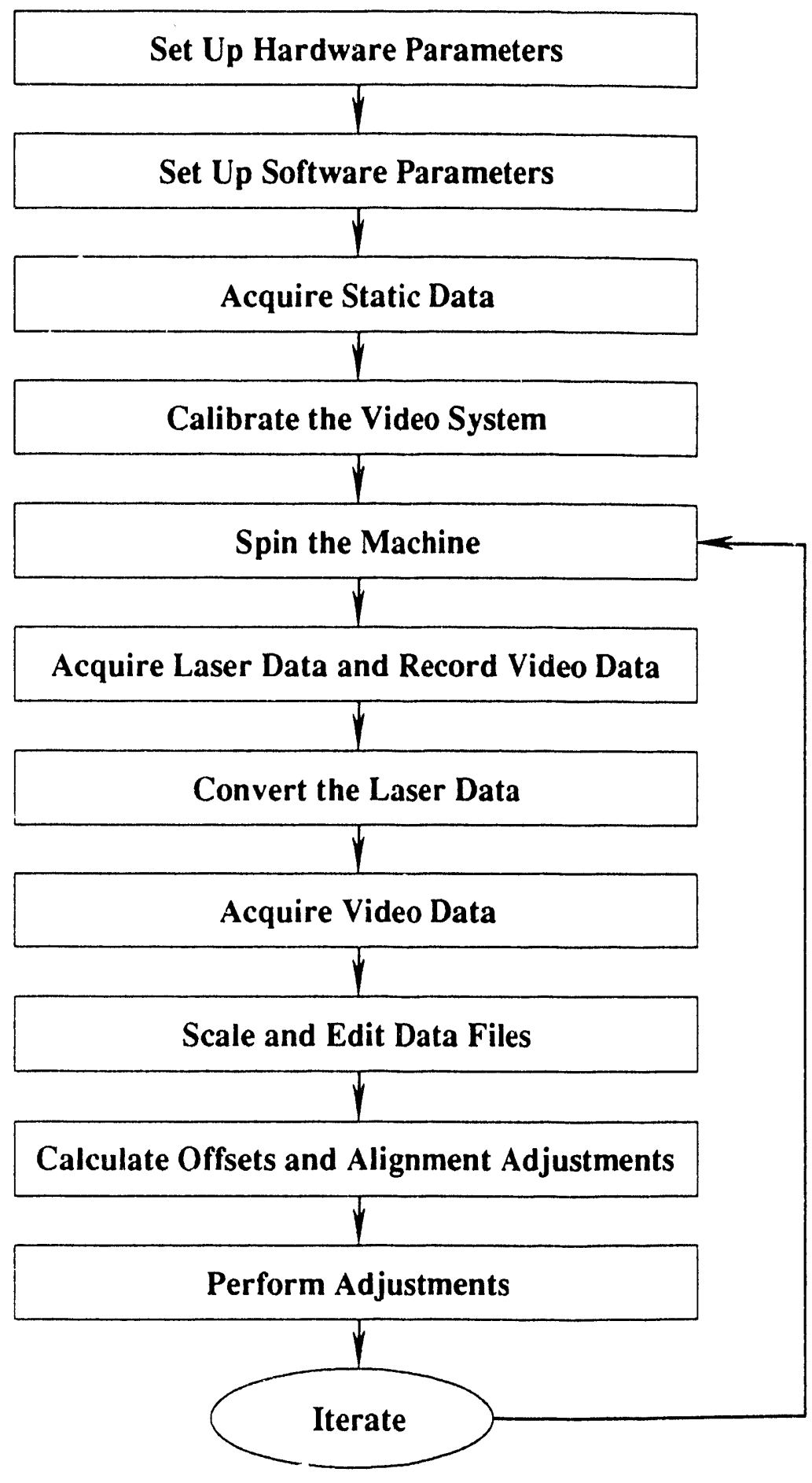

Figurr : 0. Schematic of Alignment Process 
familiar with the video data analysis system to set these parameters properly. The camera focus, test object distance, or lighting conditions should not change after these parameters are set.

The third step is to acquire another set of static video data from the test object. However, the EV commands are automated by user programs as described in Appendix B. This set of static video data is used for calibration as denoted in step four of Figure 10. A marker with a known distance must be available for video processing. Typically, this distance is the diameter of the reference marker which is a $1 / 2$ inch tooling ball for the application described herein. This known distance is equivalenced to the distance measured in the camera coordinates (with units of pixels) which spatially calibrates the video system. A calibration of the angle marker also occurs at this step. The test object and all rotating fixtures are manually oriented to align $0^{\circ}$ with the $0^{\circ}$ location of the machine. The initial orientation of the angle marker with respect to the reference marker is then calculated which calibrates the angular measurements. These first four steps need only be performed once for a given alignment, however the remaining steps are all repeated for each iteration required for alignment.

The fifth step of Figure 10 requires the user to spin the test object using the mass properties machine and subsequently acquire data during rotation. Diagnostic software is available on the POI50-Z which allows the user to spin the object at a desired speed, however numerous safeguards are bypassed on the machine using this feature. Therefore, it is very important to realize that the test object is neither aligned nor balanced and a minimum speed must be used. Typically $12 \mathrm{rpm}$ is used as the target speed for this step, which is considered safe for all conditions.

The laser displacement sensors can only be effectively adjusted while the object is rotating. Provisions are made in the software to allow the output of the laser sensors to be checked before acquiring data from the rotating object. This allows position of the laser sensors and hardware settings to be adjusted as needed. Reflectivity of the surface is detrimental to usage of the laser sensors and dull, anodized, or nonmetallic surfaces work best. The previous data collection of static data required only video information and the video processed images were sent directly to the computer. However, the computer cannot acquire data from the laser sensors and the video camera simultaneously. Therefore, the laser data is sent directly to the computer and the video data is recorded on video tape to satisfy step six of Figure 10. The user-generated signal which initiates laser data acquisition also dubs an audio tone on the video tape. This tone is later used to initiate video data acquisition from the tape and serves to reference the laser and video data to a common starting point. Step seven of Figure 10 converts the laser data which has just been acquired into a form which can be read into the EV software. The video data is then processed and read into the EV system from the video tape in step eight.

Several processing functions must be performed on the data (step nine) before calculations can be attempted (step ten). The video data must be scaled and extraneous data paths removed from consideration. The $x$ and $y$ displacements of the reference marker and the angular orientation as calculated by the angle marker are available as functions of time. The laser data must also be properly scaled and displacements of the object surface from each sensor are also available as functions for time.

The calculations of the offsets and required adjustments are made in step ten using the data generated in step nine. The top offset or misalignment of the reference axis at the upper measurement point is calculated using procedures developed in Appendix C. Then the bottom offset or misalignment of the reference axis at the lower measurement point is calculated using techniques described in Appendix D. Certain geometric errors result from using the procedures of Appendix D on conical shaped objects. Although these errors are not defeating and are handled quite well with iteration, a more efficient means of calculating the bottom offsets are proposed in Appendix E. After the top and bottom offsets are calculated, the user is prompted for the height of the upper measurements, the height of the lower measurements, and the distance from the machine spin axis to the contact points of the linear gauges $(\mathrm{z}$ adjustment moment arm) which measure $z$ deflection for tilt adjustment (see Figure 9). The necessary adjustments to bring the object into alignment are then calculated and presented to the user. It should be noted that the $z$ adjustment moment arm is only known before the first adjustment is made (assuming the 
user has aligned the tilt-translation table without the cone using traditional techniques). Each time an adjustment is made a small error is added to this moment arm measurement. Also the same moment arm is used for both $\mathrm{x}$ and $\mathrm{y}$ tilt adjustments. These are also small errors which are handled by iteration which could be removed for a more efficient process.

The user actually performs the requested adjustments in step 11 of Figure 10. As seen in Figure 9, linear gauges for all four adjustment directions are placed on the tilt-translation table and then zeroed. Then, the user loosens all locking devices on the tilt-translation table. Then all four adjustments are made sequentially and iteratively while being monitored simultaneously. This was found to be necessary since a great deal of mechanical cross-talk exists between the four adjustments. The locking devices are tightened and the linear gauges are monitored for changes to the adjustments. After the table is locked in place, the linear gauges are removid and the user can iterate on the process by returning to step five of Figure 10.

The entire alignment process has been detailed in this section, to give the reader a global view of the entire procedure.

\section{RESULTS}

This section presents actual data from the aligriment of a typical test item as seen in Figure 4 . The alignment procedure was performed four times and three adjustments were performed. Three input parameters are requested from the user to calculate the required adjustments. The same parameters were used for each iteration. The first requested parameter is the height of the upper measurement (video) above the ball bearing of the tilt-translation table ( 55.469 inches was used). The second requested parameter was the height of the lower measurement (laser) above the ball bearing of the tilt-translation table (5.635 inches was used). The final parameter was the distance from the center of rotation to the linear gauge measurement points for the tilt adjustments (a constant 9.625 inches was used). This parameter can only be measured when the fixture has been aligned with the spin axis before the test object is mounted. As mentioned previously, this parameter changes with each adjustment and it changes differently in the $0^{\circ}$ and $90^{\circ}$ directions. However, these changes are small and are handled effectively by iteration.

Table 1 contains the results of these four iterations of the alignment procedure. The top offsets as measured by the video system are given as well as the bottom offsets. These parameters provide information as to the actual alignment of the object. The $X$ and $Y$ translation parameters provide the user with the actual translation adjustments needed to brir $\mathrm{g}$ the object into alignment. The $\mathrm{X}$ and $\mathrm{Y}$ tilt parameters provide the user the actual vertical adjustments (as measured at the edge of the fixture) needed to bring the object into alignment. All four adjustments should be applied and monitored simultaneously. Constant improvement in the alignment can be observed until iteration \#4. The lower limit of the alignment and adjustment measurements is estimated as .0001 inches. This limit appears to result from the uncertainties in the adjustment of position rather than the measurement of position. Therefore any adjustment which is less than .0001 is not attempted. The entire alignment process required less than two hours.

Graphical feedback is provided to the user on each iteration. This information can be used to verify that the offsets are being calculated properly and to obtain a visual indication of the current status of the alignment. Figure 11 shows the $\mathrm{x}$ and $\mathrm{y}$ direction video data from the first iteration of the alignment process. The test data is represented by the dotted line while the solid line provides the synthesis using the calculated offsets for the video data. The frequency plots show that the fundamental rotation frequency of $.2 \mathrm{~Hz}$ dominates the data set. The signal-to-noise ratio is very high as the object is out of alignment. And finally, the data is fit very well by the synthesis, which provides confidence that the offsets have been calculated properly. Figure 12 provides the same information for the laser sensors. 


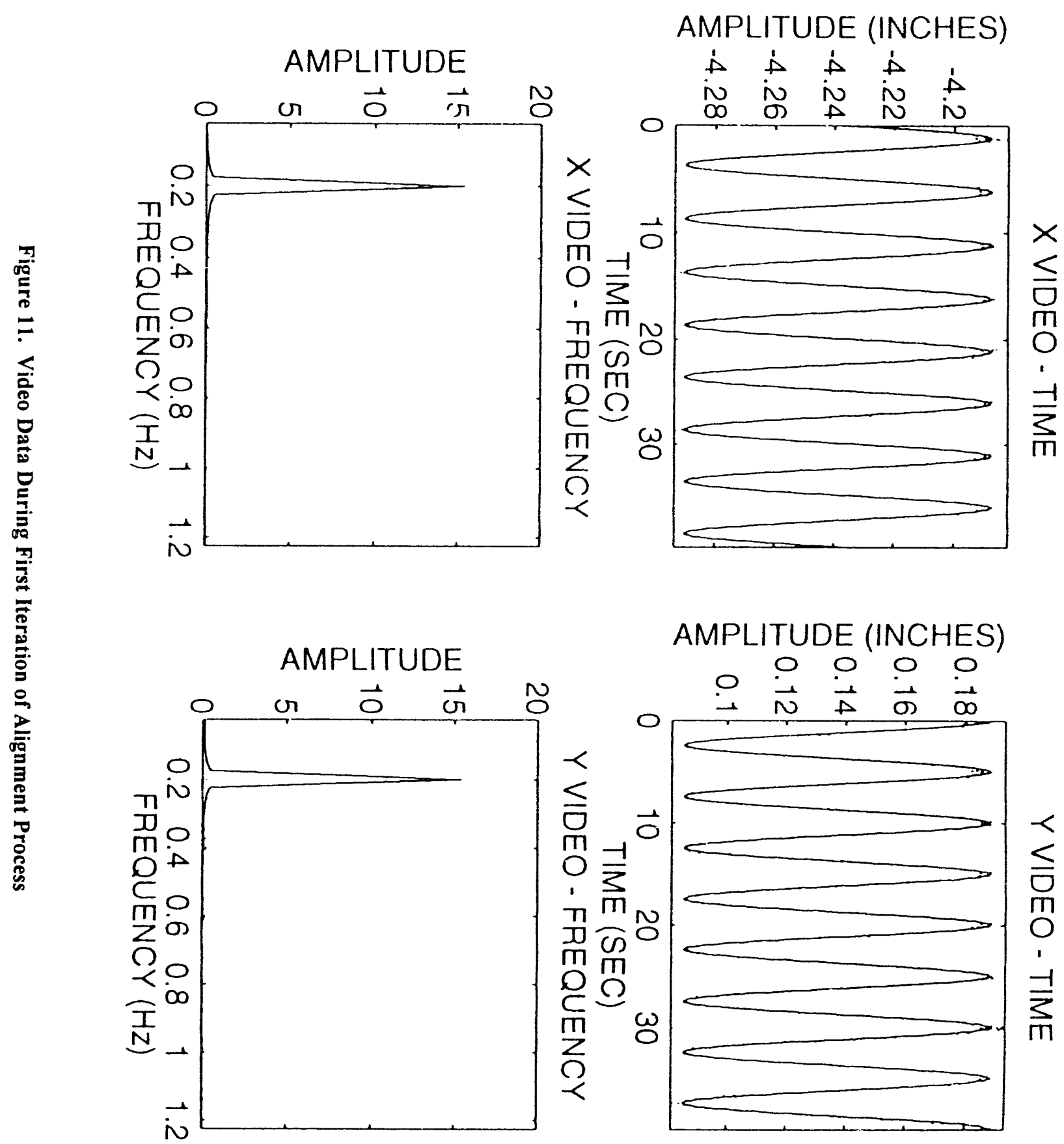



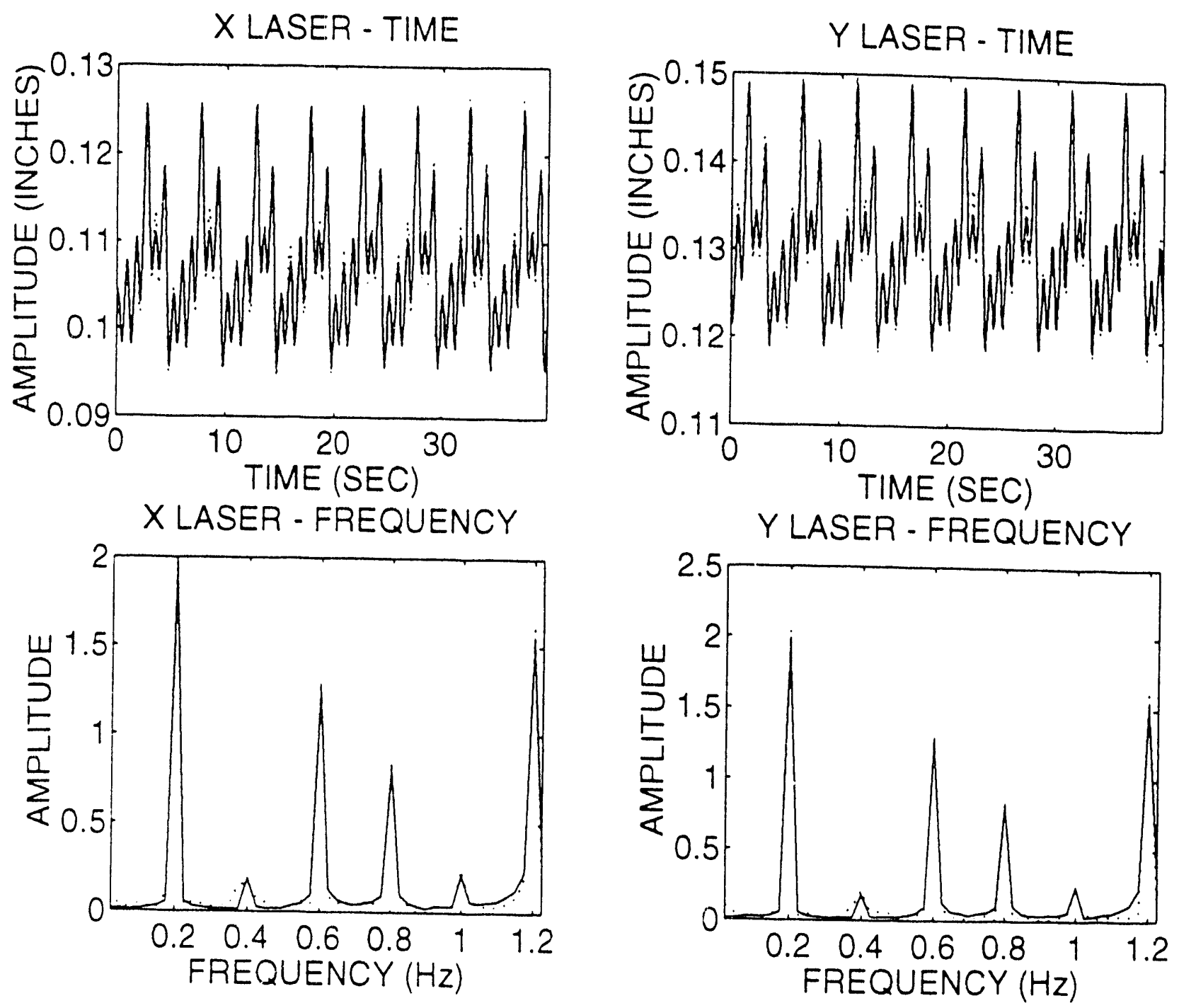

Figure 12. Laser Data During First Iteration of Alignment Process 
The response is not dominated by the fundamental rotation frequency as is the video data, but contains substantial response at the higher harmonics of the rotation frequency. These harmonics are due to misalignment of the object, misshapen surface of the object, and geometric considerations which arise from measuring the surface of the object. Appendix D contains more information on the source of these harmonics. Again, the fit to the original data provides confidence that the calculated offsets are appropriate.

Table 1. Measured Offsets and Calculated Alignment Adjustments for Each Iteration

\begin{tabular}{|l|c|c|c|c|}
\hline (Units - inches) & Iteration \#1 & Iteration \#2 & Iteration \#3 & Iteration \#4 \\
\hline Upper X Offset & -.03011 & .00996 & -.00031 & .00104 \\
\hline Upper Y Offset & .04161 & -.01413 & .00239 & .00061 \\
\hline Lower X Offset & -.00117 & -.00097 & -.00008 & .00016 \\
\hline Lower Y Offset & -.00653 & .00143 & -.00018 & .00056 \\
\hline X Translation & -.00210 & .00221 & .00006 & -.00006 \\
\hline Y Translation & .01197 & -.00319 & .00047 & -.00055 \\
\hline X Tilt & .00559 & -.00211 & .00004 & -.00017 \\
\hline Y Tilt & -.00930 & .00301 & -.00050 & -.00001 \\
\hline
\end{tabular}

Figure 13 provides the data from the fourth iteration, after alignment. Although the data fit is reasonable, the signal-to-noise ratio is very low. This is an indication that the object is very well aligned, since the sinusoidal signal results from misalignment. Figure 14 contains the laser data from the fourth iteration. The absence of the strong fundamental frequency is an indication that the object has be aligned well.

Experience has shown that four iterations, as shown in this section, is a reasonable number to expect when aligning an object to less than .001 inches top and bottom. It is also common place to stop making adjustments when changes of the magnitude of a few ten-thousandths of an inch are requested. Also, the user can effectively use the graphical outputs to check the progress of the alignment process.

\section{CONCLUSIONS AND RECOMMENDATIONS}

Experience has shown that the alignment system can align objects to within the adjustment capabilities of the operator and the fixturing. This translates into an alignment of less than .001 inches top and bottom with requested adjustments of a few ten-thousands of an inch for the application reported here. The system also defines a reference axis when the geometry of the test part does not.

The typical time required to perform the alignment is quite reasonable. This is due not only to the robust nature of the measurements but also to the rapid learning curve of the procedures. The procedure does not require any contact measurements on the object and is therefore highly adaptable to different types of test objects, especially very compliant objects. The procedure is also based on a modular set of user programs which further add to the flexibility of the alignment procedure. Therefore, the alignment system is robust, flexible, and accurate.

Several incremental improvements have been suggested in this report. These improvements rnay make the process more efficient while maintaining or improving the useful features mentioned above. The adjustment calculations can be improved by constantly updating the moment arm distance used to calculate the tilt adjustments. These adjustment calculations can be further refined by accounting for discrepancies which arise in measuring the surface of a tilted conical object. Appendix F provides a full derivation of the required upgrade. It would also be useful to develop software which automated calibration of the laser displacement sensors. 

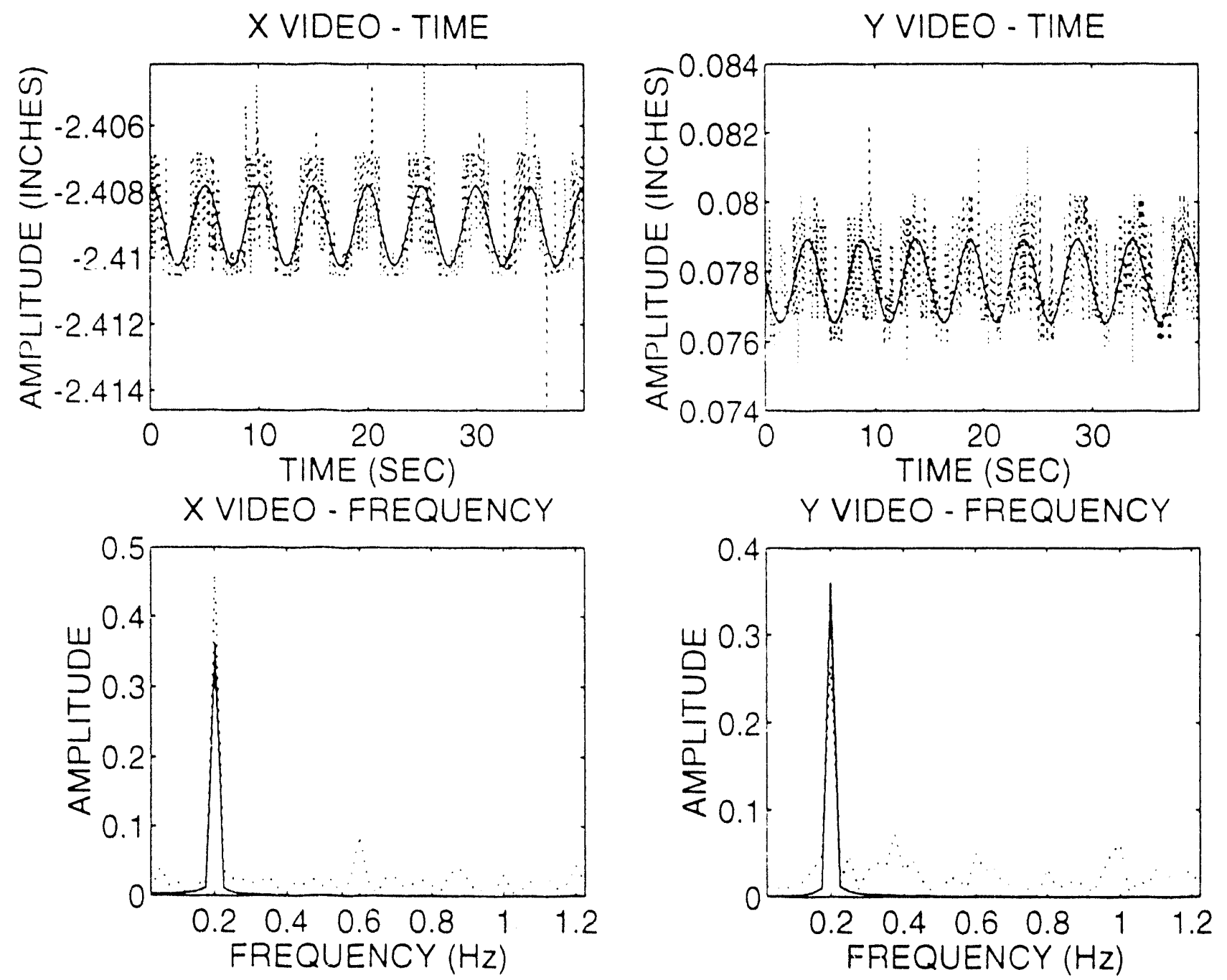

Figure 13. Video Data During Final Iteration of Alignment Process 

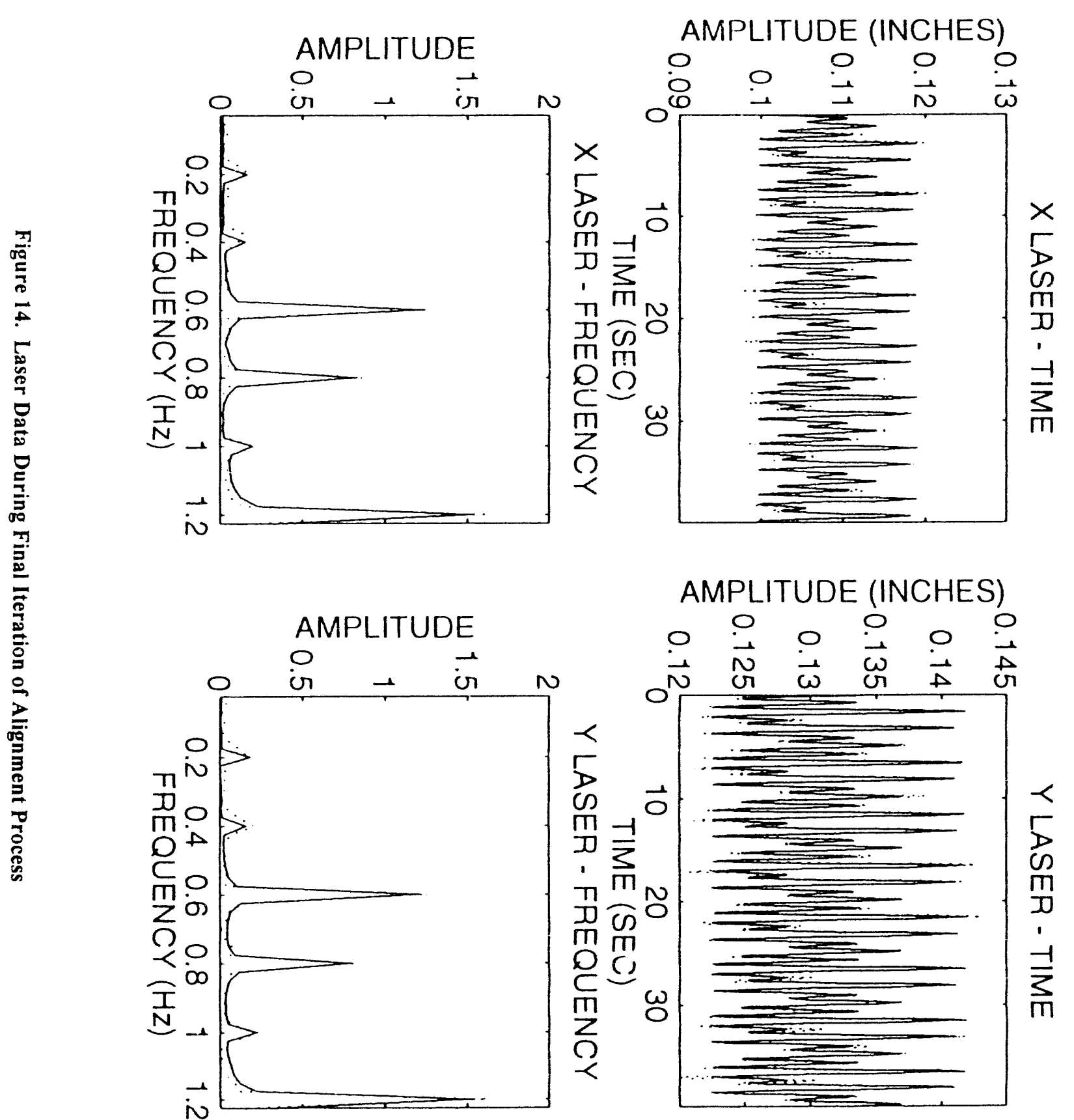
APPENDIX A

INSTRUCTIONS FOR MASS PROPERTIES MEASUREMENTS AND ALIGNMENT

A-1 


\section{APPENDIX A \\ INSTRUCTIONS FOR MASS PROPERTIES MEASUREMENTS AND ALIGNMENT}

\section{Mass Properties Measurements Procedures}

The following is the procedure for determining mass properties on the Space Electronics Model POI-50Z Spin Balancing Machine and the Model KSR330-16 Moment of Inertia Instrument:

1. Initiate all procedures required to prepare the spin balance machine, POI-50Z, for dynamic balancing of the test item. The POI-50Z equipment should be used as described in the instruction manual entitled "Model POI-50Z Operating Instructions, Serial No. 2126." DO NOT UNDER ANY CONDITIONS EXPERIMENT WITH YOUR OWN OPERATIONAL TECHNIQUES!

2. Measure the tare of the POI-50Z spin mounting table.

3. Determine the weight of the tilttranslation fixture.

4. Install tilt/translation table on the POI-50Z instrument.

5. Indicate tilt and translation to "zero" settings with respect to the vertical spin axis of the POI-50Z instrument.

6. Determine the weight of any additional fixturing.

7. Install the additional fixturing and indicate with respect to the spin axis of the POI-50Z instrument.

8. Determine the weight of the test item.

9. Install the test item and align with respect to the spin axis of the POI-50Z instrument. This alignment may be accomplished with the non-contact method described in the body of this report.

10. Remove the test object in such a manner that all fixture hardware can be replaced in the exact position it occupied while the test item was being aligned.

11. Balance the tilt/translation table and all additional fixturing in the aligned position.

12. Determine the tare of the balanced POI-50Z spin mounting table, tilt/translation table, and all additional fixturing.

13. Replace the test part and conduct POI measurements following procedures prompted by the computer. Run POI calculations and determine correction weights required.

14. Install correction weights on the test object without removal from POI instrument if possible. If not possible, remove from POI instrument and install correction weights.

15. If correction weights are installed without removal from POI instrument, then rerun POI measurement to determine effects on POI due to correction weights. Repeat procedure until POI values are acceptable. Skip to step number 17.

16. If test object has to be removed for addition of correction weights, remove the test object in such a manner that all fixture hardware can be replaced in the exact position it occupied while measurements were being made. Add weights and reinstall in the exact position on the POI 
instrument and fixturing. Determine effect of correction weights. Repeat process until POI values are acceptable.

17. Remove test object carefully in a manner that all fixturing hardware can be replaced in the exact location that it was removed from.

18. Install vertical fixture on KSR330-16 Moment of Inertia Instrument. As necessary, align fixturing and test items using the same techniques as above.

19. Determine "CG" and "MOI" properties on KSR330-16 instrument for both air and helium atmosphere as described in the "Mass Properties Instrument, Model KSR330-16, Instruction Manual" and as described in Appendix 4: Helium Chambers.

20. If mass properties are desired in vacuum, then the technique for $\mathrm{MOI}$ in vacuum for test parts measured in air and helium is described in SAWE technical paper No. 2024 entitled "Using Helium to Predict the Mass Properties of an Object in the Vacuum of Space." This paper was written and presented at the Annual SAWE Conference in San Diego, California, 17-19 May, 1991. This procedure was updated and submitted to Sandia Corp. on May 12, 1992.

21. As an important reminder, do not attempt to measure any mass properties on either instrument unless a tare value measurement is obtained prior to mounting test part.

22. Steps $18,19,20$, and 21 are repeated using the horizontal fixture for the desired test part orientations.

23. The importance of accurate fixturing cannot be over-stressed. The measurement of mass properties can never be any more accurate than the quality of the fixturing.

\section{Motion Analysis Alignment Procedure for the Spin Balance Machine}

March 24, 1993

\section{SET UP COMPUTER}

Boot up computer by turning ON the power button on the Samsung monitor.

Menu will come up on screen.

Type $l$ and press Enter for menu item 1, VP1 10 checklist.

\section{EV SOFTWARE CHECK, CALIBRATION AND INITIAL ANGULAR BEARING}

Type 2 and press Enter

Type vide, should be set to 40 secs $<$ cr $><$ cr $>$.

Push the remote button.

Type $p l o t$ to verify data quality after acquisition.

Type cent and assure two paths by adjusting the parameters

(typical values - 224200 )

Type path and assure two paths by adjusting the parameters.

(typical values - 1050210 )

Calibration

Type spli to further check the "path" results.

Set table to align the 0 degree direction with the ubjeci's 0 .

At "EV:" type (a) cal $<<c r\rangle$.

At "Ready for Video Input", push remote button to take video data.

(take 40 seconds of video data)

After video file plot, $<\mathrm{cr}>$. 
After centroid plot, $<\mathrm{cr}>$.

Record the reference and angle path numbers for the next procedure.

After path file plot, $<$ cr $>$.

Use mouse to enter calibration measurements.

If the scaling procedure fails, type scale and try again.

Initial Angular Bearing

At "EV:", type @staticl (reference path) (angle path) $<\mathrm{cr}><\mathrm{cr}>$.

At "list:", <cr>.

At "EV:", type list/lp $<\mathrm{cr}><\mathrm{cr}>$ for hard copy.

At "EV:", type quit

\section{PREPARE TO ACQUIRE LASER DATA AND RECORD VIDEO DATA}

Use POI Diagnostic s program to start POI rotating at $12 \mathrm{rpm}$ (SE).

If ADS system does not perform properly, reboot computer with Reset button.

Type 3 and press Enter for menu item 3.

Type $e$ for execute.

Type $l$ for local.

At "File?", type adsl.

Observe the traces to assure quality data.

Type a $<$ cr $>$ when satisfied.

Acquire Data

Insert tape (VCR).

Rewind tape, reset counter, or note counter setting (VCR).

Read both:

Push the "REC" buttons simultaneously on the remote control (VCR).

Record for at least 10 seconds and then push the remote switch.

After the "beep", type $q$ to quit (do not use $<\mathrm{cr}>$ ).

Push the "STOP" button (VCR).

Read both:

Push the "PLAY" button and use reverse knob to rewind tape.

Push the "STOP" button (VCR).

Toggle the POI machine off (SE).

\section{CONVERT LASER DATA}

Type 4 and press Enter for menu item 4 .

Type in filename ads0l.ana.

Assure a successful conversion to ads01.ats with the following:

Data collection interval $=40.0000 \mathrm{sec}$.

Sampling rate $=300 \mathrm{~Hz}$.

Interval $=20$.

$\#$ of channels $=2$.

\section{COLLECT VIDEO DATA AND EDIT VIDEO AND LASER FILES}

Type 5 and press Enter for menu item 5 .

Read all:

At "EV:" type (a)image2 $\langle c r\rangle$.

At "Ready for Video Input" begin VCR with "PLAY" button (VCR).

Observe the recorded image at the computer screen or monitor.

Adjust the threshold knob for the proper image.

After data acquisition, push the "STOP" button (VCR).

After video file plot enter $\mathrm{a}<\mathrm{cr}>$. 
After centroid file plot enter $\mathrm{a}<\mathrm{cr}>$.

Record the reference and angle path numbers for the next procedure.

After path file plot, enter $\mathrm{a}<\mathrm{cr}>$.

After $x$ direction plot, enter $a<c r>$.

After y direction plot enter $\mathrm{a}<\mathrm{cr}>$.

Edit Video and Laser Files

At "EV:" type (a)editl (reference path) (angle path) (static bearing), <cr>.

Note: static bearing is the "Average Value "from the STATICl listing.

At "EV:" type list/re v.ans, <cr>.

At "list:", type v.dat, <cr>

At "EV:", type quit, <cr>.

\section{CALCULATE OFFSETS AND ALIGNMENT ADJUSTMENTS}

Type 6 and press Enter for menu item 6 .

At ">>", type calcl, <cr>.

Verify video data fit and enter $\mathrm{a}<\mathrm{cr}>$.

Verify laser data fit and enter $a<c r>$.

At "input upper height", input the height of the center of the tooling ball and enter $a<c r>$.

At "input lower height", input the height of the laser heads and enter a $<\mathrm{cr}>$.

At "input moment arm", input the length of the moment arm and enter a $<\mathrm{cr}>$.

At ">>", hit the "Print Screen" button.

After printing type quit.

\section{ITERATE}

Loop to 3, Acquire Data 
APPENDIX B

STRUCTURE OF SOFTWARE

B-1 


\title{
APPENDIX B
}

\section{S'TRUCTURE OF SOFTWARE}

Hardware settings, software packages, user programs, commands, data files, and data variables will be denoted by capital letters in this report.

\section{ALIGNMENT MENU}

The software to perform the alignment has been packaged into a menu driven format. This menu is produced by the DOS file MENU.COM and reproduced below in Figure B-1. Step-by-step instructions for performing the procedures in this menu are included in Appendix A.

\section{MOTION ANALYSIS ALIGNMENT PROCEDURES}

\author{
1. VP110 Checklist \\ 2. EV Software Check, Calibration, and Initial Angular Bearing \\ 3. Prepare to Take Laser Data and Acquire Data (ADS) \\ 4. Convert Laser Data \\ 5. Collect Video Data and Edit Video and Laser Files \\ 6. Calculate Offsets and Alignment Adjustments (Matlab) \\ Iterate - Goto Step 3
}

Type the number you want and press ENTER:

Figure B-1. Reproduction of Alignment Procedures Menu

The first menu item is a listing of hardware settings to be made before acquiring data. The second menu selection allows the user to check software parameters needed for the alignment procedure and acquire data to calibrate the video system displacement and angular orientation measurements. The third step requires the user to spin the machine slowly (typically $12 \mathrm{rpm}$ is used) and acquire analog data from the laser displacement sensors for 40 seconds at 15 samples per second. Also at this step, the user records video data on tape along with an audio tone which will later be used to initiate video data collection and synchronize with the laser data. The fourth selection converts the laser data into a form which can be combined with the video data. The fifth menu item allows the user to acquire video data from the tape. Several coordinate changes and scalings of the video and laser data also occur in the fifth step. The final result of this selection is an ASCII disk file which contains all video and laser data scaled to inches of displacement as well as the angular orientation of the test object in degrees. The important mathematical calculations occur in the sixth step after the data file is edited and loaded in Matlab. The user must enter data on the height of the top and bottom measurements and a moment arm for vertical adjustments.

Calculations of the offset at the top measurement (video), offset at the bottom measurement (laser), and the required alignment adjustments are then performed. The user is then instructed to loop to step three if another iteration is required.

\section{VP110 CHECKLIST}

The DOS batch file 1.BAT is called when menu selection 1 is entered. The listing is provided below and is seen to call the command CHECK.COM which displays a checklist of hardware settings for the VP110 processor. Figure B-2 includes a reproduction of the VP110 Checklist menu. The reader is referred to 
the Users' Manual for the VP110 processor for detailed descriptions of these settings [7]. However, a short description is provided in this section. The first step instructs the user to set the frame rate to 15 fields per second for video data collection. The laser analog data will be collected at this same rate. The second step is an instruction to allow the monitor to display the video picture at the full rate for ease of viewing. Video data acquisition will be initiated with a standard audio tone labeled event 1 and this setting is requested in step three.

\section{VP110 CHECKLIST}

1. Set "Frame Rate" to 15

2. Turn ON "Full Rate" light

3. Turn ON "Event 1" light

4. Turn ON "Window On" light

5. Make sure "Clear Overflow" light is off

6. Set the "Threshold" for a good image (It may help to toggle the "Video" light off)

Figure B-2. Reproduction of VP110 Checklist Menu

Although the greatest resolution is obtained when the usable scene fills the field of view, this is typically not the case. A windowing feature is available to limit the amount of unwanted scene and step four suggests that the user initiate this capability. The user is referred to the VP110 manuals for instructions on setting this window [7]. If a data collection process is interrupted or too much data is passed to the computer an "overflow" occurs and it must be "cleared" before data acquisition can be resumed. Step five reminds the user to check for this situation and correct it. The most important hardware setting is requested in step six. The grey scale threshold setting determines the quality of the video data which will be recorded by the computer. The user should constantly be aware of the data quality and adjust the threshold setting as needed. This is especially critical when transferring data from video tape. It is suggested that the user turn off the video image so that the binary edges can be viewed easily. Figure B-3 provides a close-up view of the video processor with the correct settings.

\section{Listing of 1.BAT}

echo off

cls

check.com

pause

menu.com

\section{EV SOFTWARE CHECK, CALIBRATION, AND INITIAL ÁNGULAR BEARING}

\section{EV Software Check}

Menu selection two runs the file 2.BAT which is listed below. The important function of 2.BAT is to call the EV software package. The user should then refer to the instructions of Appendix A for as list of the steps to follow for setting the EV software parameters by manually executing each step of the video analysis (VIDE, PLOT, CENT, PATH, and SPLI) with a dummy set of data. This is a critical step which requires some experience and familiarity with the software to perform [4]. Typical values for the software parameters are provided in the instructions as a starting point. For large pixel number outlines, it is recommended that data be sampled at 15 frames per second to avoid overflows. Six hundred frames of data (40 seconds) is recommended. 


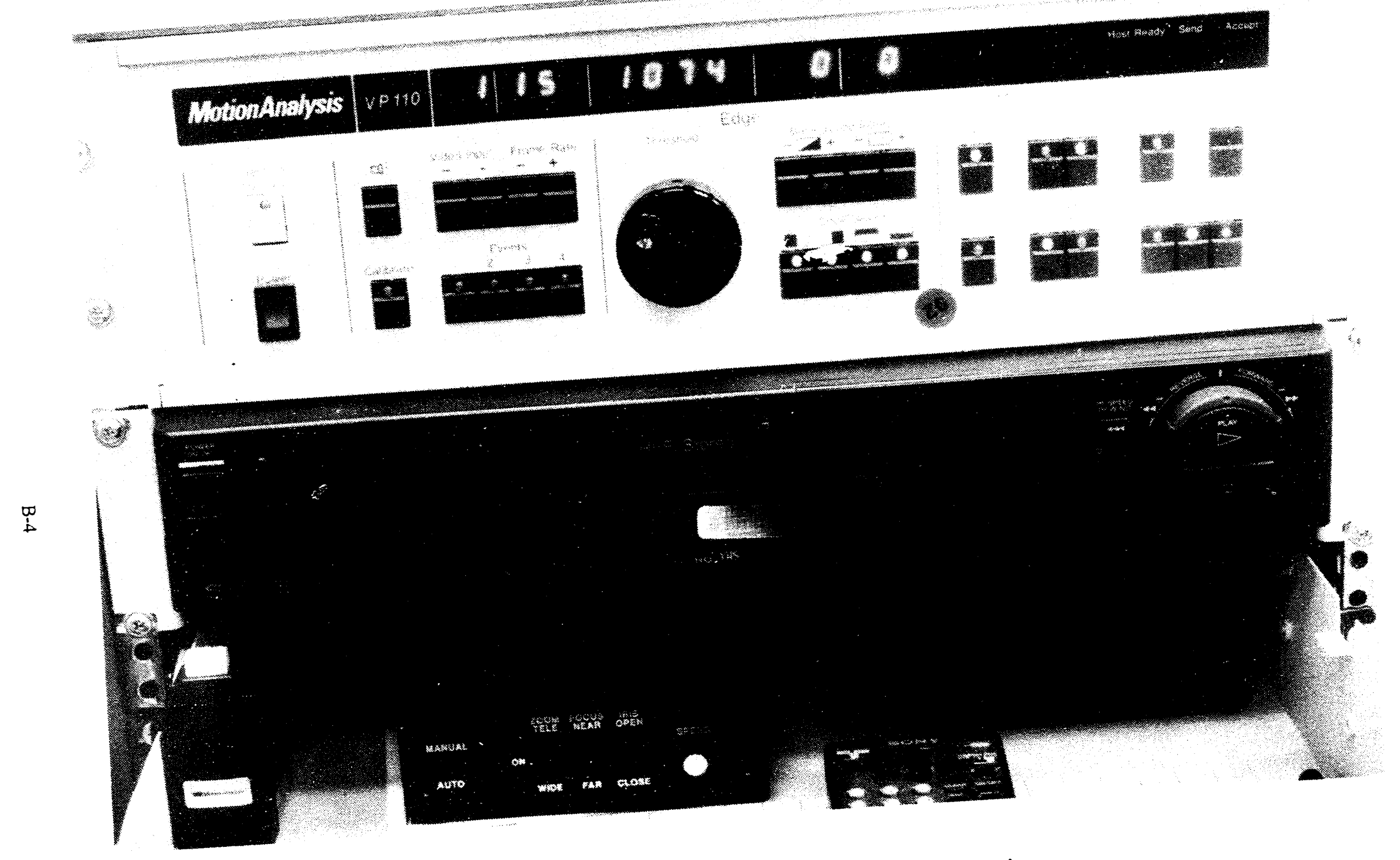

Figure B-3. Close-up View of Video Processor with Correct Settings 


\section{Listing of 2.BAT}

echo off

cls

call ev

menu.com

\section{Calibration - Program CAL2.EV}

An Expertvision user program is provided to perform initial calibration of the video system and the listing is included below. CAL2.EV provides the user with a set of commands to acquire static video data and subsequently calculate the distance calibration. The user must have previously set all video software parameters as discussed in the previous paragraph. The video filename is set to be V.VID and data acquisition from live video is initiated with the EVENT 1 signal. After the video data is acquired with the VIDE/GO command, a plot is drawn and a carriage return is required to continue. The centroids of the markers in each video frame are calculated with CENT/GO and a carriage return is required (output file V.CEN). The PATH/GO command connects the centroids in time and plots the results including the path numbers (output file - V.PAT). The user must record the path number of the reference axis path and the angle marker path. These parameters are input to the next program.

The final function of CAL2 is to calibrate the distance scale. The user should already have a known distance in the field of view. Typically, this is the diameter of the reference marker. The SCALE command performs the calibration. The user is asked to define the known distance with the mouse and input the actual length. The user is then asked to provide the units. This command has a high probability of failing. However, the user can type in "scale" immediately upon return of the prompt to rerun the command.

\section{Listing of CAL2.EV}

$\mathrm{crt} / \mathrm{cl} / \mathrm{ec} / \mathrm{sc}$; clear crt, enable echo, and enable scrolling

; FILE $=$ IUSR \ALIGN\CAL2.EV

,

; INVOCATION: "@cal2"

vide/go v.vid

plot

pause/cr

cent/go

pause/cr

path/go

plot/nu .pat

pause/cr

scale .vid

\section{Initial Angular Bearing - Program STATIC1.EV}

The user program STATICI.EV calculates the initial angular orientation of the angle marker with respect to the reference axis marker. The user must input the path numbers for the reference and angle path as mentioned before. Also the user must have the body $0^{\circ}$ location lined up with the machine $0^{\circ}$ location. The angular relationship between all the paths in the file with respect to the reference path number is calculated with the BEAR command (output file - V.BER). The EDIT/SA/IN command edits the resulting file to remove all bearings except that of the angle path. The mean of the angular path bearings 
are calculated with the STAT command and the results are listed to the screen (output file - V.STA). The user should record this mear: value of the static bearing of list the file V.STA to the printer.

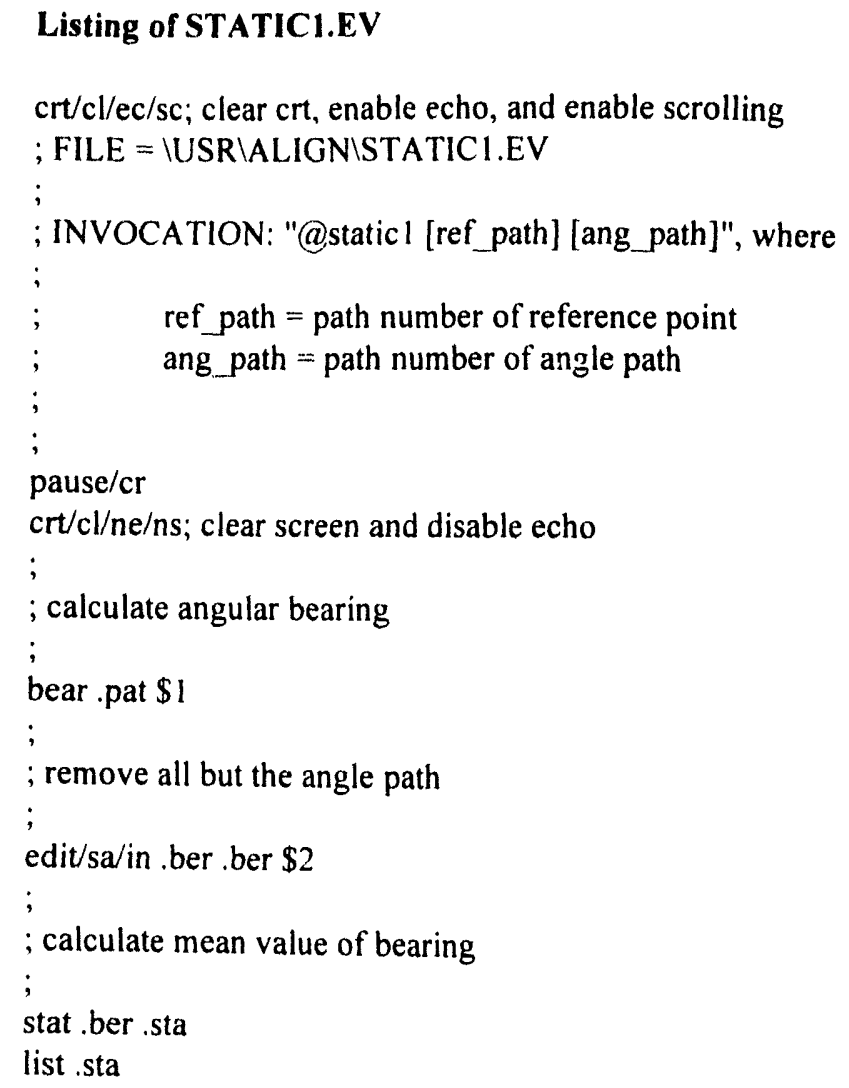

PREPARE TO TAKE LASER DATA AND ACQUIRE DATA (ADS)

\section{Collect Laser Data - Program ADS1.ADS}

Menu selection three run the file 3.BAT which is listed below. The primary function of this file is to enter the ADS software which can manually be entered by typing "ADS". A user file for the ADS system, ADSI.ADS has been written to automate collection of the laser data. The user file is executed by typing "E" for EXECUTE and "L" for LOCAL. The user file name is then entered without extension
(ADS1). This user file performs (ADS1). This user file performs several functions in the SETUP mode. First, input channels one and two channel 1 is defined to be the "ylaser" respectively. Second, the scope channels are defined. Scope with a scope gain of one. Scope channels scope gain of one. Scope channel 2 is defined to be ylaser of two. The duration of data channels three and four are defined as xlaser and ylaser with scope gains data points per second and the interval is to be 40 seconds. The frequency of data capture is set to 300 sample and hold and to compensate the frequency is set high with a The ADS is unfortunately not lag between the collection of chate the frequency is set high with a large interval. This limits the time to 2.0. The output file nam of channel 1 and channel 2 data. And finally, the gain on the ADS card is set scope feature. Final data is collected to be ADS01.ANA. The data is then set to be previewed with the object is rotating at $12 \mathrm{rpm}$. And finally, an EVENT 1 trigger signal from the hand switch while the 


\title{
Listing of 3.BAT
}

echo off

cls

call ads

menu.com

\section{Listing of ADS1.ADS}

\author{
\# ADS1.ADS \\ \# ADS batch file to collect and acquire data \\ setup channels 1 xlaser \\ setup channels 2 ylaser \\ setup scope 1111 \\ setup scope 2121 \\ setup scope 3112 \\ setup scope 4122 \\ setup duration 40 \\ setup frequency 300 \\ setup interval 20 \\ setup gains 22 \\ file name ads \\ file trial 01 \\ collect preview \\ collect trigger \\ quit
}

\section{CONVERT LASER DATA}

The laser output file, ADS01.ANA, must be converted to a form that the EV software can read. Once in DOS, the user can type in "ANAREAD" and provide the full output file name. However menu selection four uses the file 4.BAT to automate this step. As seen below, a directory listing is provided which allows the user to check for existence, size, and date of the output file. After a carriage return, the command ANAREAD is executed and the user enters the output file name. The file ADS01.ATS is created and a description of the data in the file is printed. The user is referred to the instructions in Appendix A to check this description.

\section{Listing of 4.BAT}

echo off

dir *.ana

pause

anaread

pause

menu.com 


\section{COLLECT VIDEO DATA AND EDIT VIDEO AND LASER FILES}

\section{Collect Video Data - Program IMAGE2.EV}

The user can reenter the EV system by typing "EV", however the DOS file 5.BAT is provided to automate this step. The EVENT 1 signal used to trigger the laser data collection is also used to trigger video data collection for the appropriate time matching. Forty seconds of data sampled 15 times per second should be used to synchronize with the laser data. An EV user program file called IMAGEI.EV has been written to automate vic' o data collection. The data analysis parameters should be set beforehand, as with the static data collection. This file collects video data, centroids the marker outlines, and links the centroids in time to form paths as the previously mentioned CAL2.EV user file did and uses the same output file names. The user must again record the reference path number and the angle path number to input to the next program. The path file is split into a file containing $x$ coordinates (V.PAX) and a file containing y coordinates (V.PAY) and these are plotted. This allows the user to verify the quality of the data as the displacements should be pure sinusoids.

\section{Listing of 5.BAT}

echo off

cls

call ev

menu.com

\section{Listing of IMAGE2.EV}

$\mathrm{crt} / \mathrm{cl} / \mathrm{ec} / \mathrm{sc}$; clear crt, enable echo, and enable scrolling

: FILE = IUSR \ALIGN IMAGE2.EV

;

; INVOCATION: "@image2"

vide/go v.vid

plot

pause/cr

cent/go

pause/cr

path/go

plot/nu.pat

pause $/ \mathrm{cr}$

spli'go

plot/nu .pax

pause/cr

$\mathrm{plot} / \mathrm{nu}$.pay

pause/cr

\section{Edit Video and Laser Files - Program EDIT1.EV}

The user must run the EV user file EDIT1.EV and provide the reference path number, angle path number, and the mean value of static bearing as determined from STATICI.EV. The angular orientation between the reference path and all other paths is calculated and the result placed in V.BER. A file of all ones is created (V.ONE) and the V.BER file is edited to remove all but the data from the angle path (output file V.THE). The initial static bearing is subtracted from the each entry in the V.THE file. The $x$ and $y$ coordinate files are edited to remove all information except that containing the reference path and the resulting files are called V.TMX and V.TMY. The coordinate system for the video camera is lef:-handed. Therefore, the y coordinate file is multiplied by -1.0 to convert to a right-handed system. 
The laser data in the output file ADS01.ATS is biased by 2047 and multiplied by the scale factor of $.00019224[5,8]$. This converts the output to inches for an ADS gain of 2.0. Equation 3 provides a mathematical description of the conversion from counts to inches.

$$
\begin{aligned}
& \# \text { inches }=\text { scale } *(\# \text { counts }-2047) \text {, } \\
& \text { where, scale }=\left(\frac{10 \text { volts }}{4096 \text { counts }}\right) *\left(\frac{1 \text { inch }}{12.7 \text { volts }}\right)=.00019224 \text {. }
\end{aligned}
$$

The laser sensors read a positive displacement away from the sensing head. This is opposite to that of the machine coordinate system. Therefore, the laser data is multiplied by -1.0 . Then the $x$ laser data is placed in ADS01.XTS and the $y$ laser data is placed in ADS01.YTS. Both data files are smoothed to remove some of the noise induced by surface irregularities. The laser data files are in the wrong format to be operated on by EV commands. Therefore, the file V.ONE is multiplied by both files to produce V.XTS and V.YTS which can be operated on. Finally, $x$ laser data, $y$ laser data, $x$ video data, and $y$ video data are collected into a single answer file called V.ANS. Several unneeded files are deleted before the user program ends. The answer file must be written into an ASCl disk file called V.DAT with the EV command LIST/RE.

\section{Listing of EDIT1.E.V}

$\mathrm{crt} / \mathrm{cl} / \mathrm{ne} / \mathrm{ns}$; clear crt, enable echo, and enable scrolling

; FILE $=$ IUSR \ALIGN\EDITI.EV

$;$

; INVOCATION: "@editl [ref_path] [ang_path] [stat_bear]", where

ref_path = path number of reference point

ang path $=$ path number of angle path

stat_bear $=$ value of static bearing angle

; calculate the angular bearing

;

bear v.pat $\$ 1$

; edit all but the reference path

edit/sa/in .ber .one $\$ 1$

add/co .one .one 1.0

;

; remove all but the angle path

;

edit/sa/in .ber .the $\$ 2$

;

; subtract static offset

;

subt/co the the $\$ 3$

;

; edit pax for reference path

edit/sa/in .pax .tmx $\$ 1$

; edit pay for reference path 


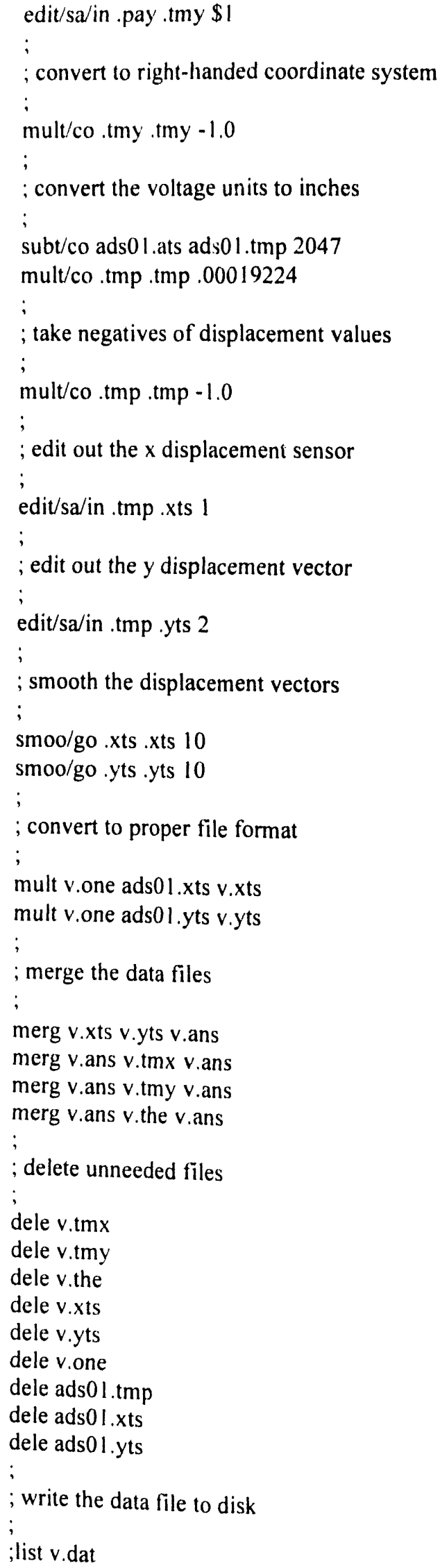




\section{CALCULATE OFFSETS AND ALIGNMENT ADJUSTMENTS (MATLAB)}

\section{Edit V.DAT - Program PROCESSI.BAT}

Menu selection six executes 6.BAT which is seen below. The primary function of this DOS file is to call a second DOS file PROCESSI.BAT. PROCESSI.BAT operates on the file V.DAT which contains all video and laser sensor data. This data can be read directly into MATLAB if the first three lines of header information are removed. As seen in the following program listing, PROCESSI.BAT uses the DOS line editor EDLINE to remove these three lines based on the commands in the file REMOVE1.TXT. The file PROCESSI.BAT then initiates MATLAB following the editing. MATLAB is used to perform the final calculation of the top and bottom offsets as well as the necessary adjustments [6].

\section{Listing of 6.BAT}

echo off

cls

call process 1

menu.com

\section{Listing of PROCESS1.BAT}

rem Batch file to calculate alignment parameters

rem

rem remove the first 3 lines of a video list file

rem

edlin v.dat $<$ removel.txt

matlab

Listing of REMOVE1.TXT

$$
\begin{aligned}
& \text { e } \\
& y
\end{aligned}
$$

\section{Numerical Calculations - Program CALC1.M}

Load the data The MATLAB m file CALC1.M performs the numerical calculation for offsets and adjustments. As seen in the following listing, the initial step is to load the data contained in V.DAT. The data is read in as a single matrix and must be broken up into the individual vectors containing the $x$ laser (LX), $y$ laser (LY), video $x$ direction (VX), video y direction (VY), angular orientation (THETA), and time (TIME) data. Six hundred data points are assumed for all data vectors.

The video camera coordinate system is not aligned with the machine coordinate system and must be rotated to properly interpret the results. For the POI-50Z, the required rotation angle was determined to be 138 degrees and is written in the code as the variable ROT. The rotation angle is converted to radians and the coordinate system rotated by the appropriate linear combination of the unrotated data. The temporary variables are then cleared.

The frequency content of each data vector is calculated with the Fast Fourier Transform (FFT) and the results placed in the vectors FLX, FLY, FVX, and FVY. The frequency domain provides some useful information for interpreting the data as will be discussed in the appendices. The vector $\mathrm{HZ}$ is filled with the frequency values which allow interpretation of the frequency data. 
Videe data analysis The upper point of the reference axis was defined by the center of the reference marker as determined by a harmonic curve fit to the video data. The theory supporting the harmonic curve fit is described in Appendix $\mathrm{C}$. The reader is referred to that information for a deeper understanding of the processing used in this section. The angular orientation data is converted to radians (RAD) and vectors of the sine $(S)$ and cosine $(C)$ values are produced. Vectors of 600 ones $(O)$ and 600 zeros $(Z)$ are then produced. A $1200 \times 4$ coefficient matrix called $A$ is filled based on the development seen in Appendix $C$. The video data files are detrended by removing the mean values and storing the results in the files BX and BY. This was found to be a numerically useful step to assure a stable solution to the least squares problem which follows. All detrended video data is collected into a single matrix $B$. The appropriate parameter vector, PV, is calculated using a least squares approach. As will be seen later, the $x$ offset is the first entry in PV and the $y$ offset is the second entry.

An analytical synthesis of the original data (BS) is calculated using the coefficient matrix A and the estimated parameters in PV as described in Appendix $r$ The mean values are added back to the data and the results stored in VXS and VYS. The FFT is used to calculate the frequency domain representation of the synthesis and the results stored in FVXS and FVYS. A set of four plots (time and frequency domain representation of the $\mathrm{x}$ and $\mathrm{y}$ direction) are provided to give the user feedback as to the quality of the data and the curve fits. The synthesized data is plotted on the same graph as the original data.

Laser data analysis This section determines the lower point of the reference axis by processing the radial information from the shape of the object as mentioned in the introduction. Appendix D describes the logic and numerical processing used to define the intersection of this axis with the plane defined by the cross-section at the laser measurement height. The first line of this section to calculate the laser fit reverses the sign on the angular orientation vector RAD. This is an important and subtle step. The video camera measures displacements as the object rotates about the spin axis. However, the laser sensor data must be interpreted as if the sensors are rotating about a stationary object in the opposite direction. The vectors $\mathrm{Cl}-\mathrm{C} 7$ and $\mathrm{S} 1-\mathrm{S} 7$ are the provided as cosines and sines of the harmonics of the angular orientation. The coefficient matrix $\mathrm{A}$ is filled as described in Appendix D. The $\mathrm{x}$ and $\mathrm{y}$ laser data is detrended by removing the mean values and storing the results in the vectors $\mathrm{BX}$ and $\mathrm{BY}$. This allows a more stable numerical problem. The output vector $B$ is filled and the parameter vector PL is subsequently estimated with a least squares procedure.

The laser synthesis, BS, is calculated from A and PL. The mean values are added back in and the synthesis results stored in LXS and LYS. The FFT is used to develop a frequency domain representation of this synthesized data and the results are stored in FLXS and FLYS. As with the video data, four plots are produced to allow the user visual feedback on the data and the synthesis.

Alignment calculation The user is asked to input three measurements necessary for the alignment calculation. The height of the center of the top measurement point from the rotation point of the tilttranslation table is first required and stored in variable ZTOP. The height of the laser sensor measurements is stored in variable ZBOT. And finally, the moment arm or distance from the spin axis to the vertical measurement point for tilt alignment adjustment is requested for variable MA. These measurements should be made as accurately as possible. However, small errors may are inevitable. The height of the bottom measurements is difficult to determine. Also, the moment arm will change as adjustments are made. However, experience has shown that these errors are small enough to be taken care of by iteration.

The slopes of the straight line curve fits ( $\mathrm{MX}$ and $\mathrm{MY}$ ) are calculated using the top and bottom offsets in the appropriate directions. The offsets (XO and YO) are then calculated. Error checks (ERRX and ERRY) are provided for diagnostics only. The slope values are scaled by the moment arm and subsequently multiplied by negative one to calculate the adjustments which actually need to be made to correct the tilt of the object. The offsets are multiplied by negative one to provide the actual numbers for the translation adjustment. And finally, the data, time, top and bottom offsets, and all four adjustmonts are printed out. Appendix E contains a further description of these calculations. 


\section{Listing of CALC1.M}

$\% \mathrm{~m}$ file to process video and laser data

$\%$

$\%$ George James 2741 October 10, 1992

$\%$

$\%$ load data

$\%$

load v.dat

$\mathrm{I}=\mathrm{v}(1: 600,4)$;

$l y=v(601: 1200,4)$;

$\mathrm{vx}=\mathrm{v}(1201: 1800,4)$;

$v y=v(1801: 2400,4)$;

theta $=\mathrm{v}(2401: 3000,4)$;

time $=\mathrm{v}(1: 600,3)$;

$\%$

$\%$ rotate camera coordinate system

$\%$

tempx $=\mathbf{v x}$;

tempy $=v y$;

rot $=138$;

rots=['The camera coordinate system is rotated by ',num2str(rot),' degrees'];

$\operatorname{disp}($ ' ')

disp(rots)

disp(' ')

pause(3)

$\operatorname{rot}=\operatorname{rot}^{*} \mathrm{pi} / 180$

$v x=$ tempx ${ }^{*} \cos ($ rot $)+$ tempy* $\sin ($ rot $)$;

$v y=-$ tempx $* \sin (r o t)+$ tempy* $\cos (r o t)$;

clear tempx tempy rot rots

$\%$

$\%$ calculate frequency values

$\%$

$\mathrm{flx}=\mathrm{fft}(\mathrm{lx})$;

fly $=f f(l y)$;

$f v x=f f t(v x)$;

$f v y=f f t(v y)$;

$\mathrm{hz}=15^{*}(0: 599)^{\prime} / 600$;

$\%$

$\%$ calculate fit to video data

$\%$

$\mathrm{rad}=$ theta $^{*}(\mathrm{pi} / 180)$;

$\mathrm{s}=\sin (\mathrm{rad})$;

$\mathrm{c}=\cos (\mathrm{rad})$;

$\mathrm{o}=$ ones $(600,1)$;

$\mathrm{z}=\mathrm{zeros}(600,1)$;

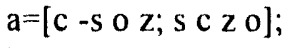

$b x=v x-m e a n(v x)$;

$b y=v y-m e a n(v y)$;

$\mathrm{b}=[\mathrm{bx} ; \mathrm{by}]$;

$\mathrm{pv}=\mathrm{a} \mid \mathrm{b}$;

$\%$

$\%$ calculate video synthesis 


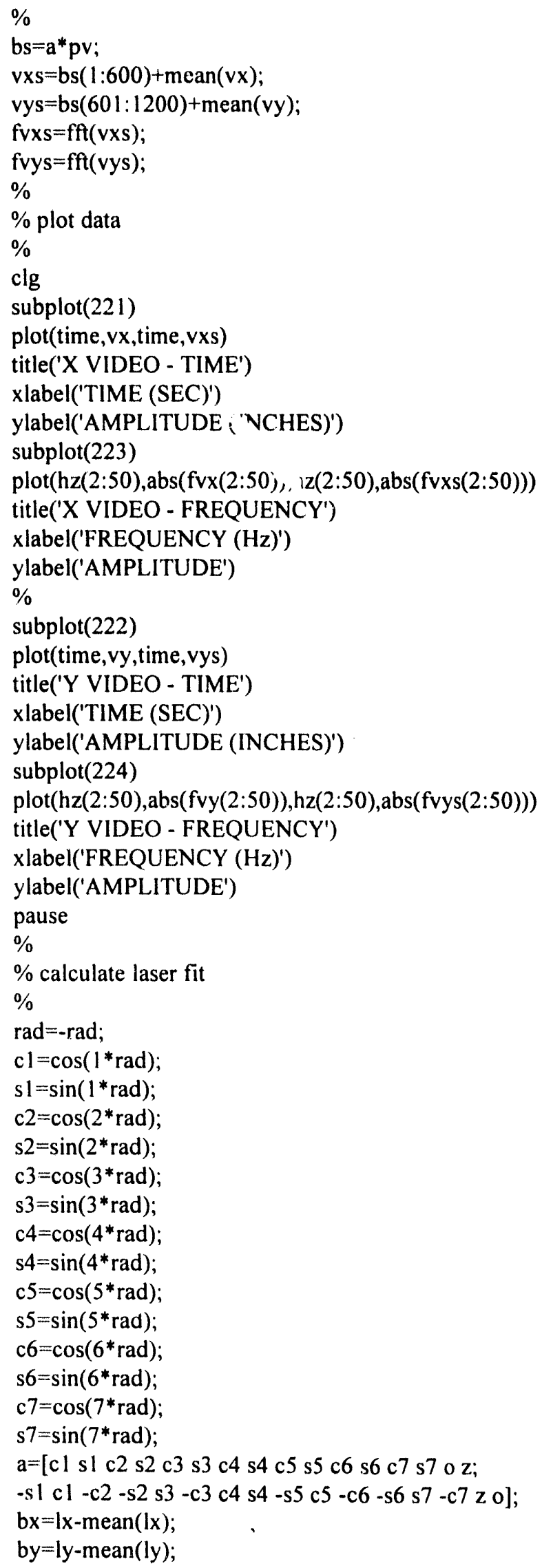




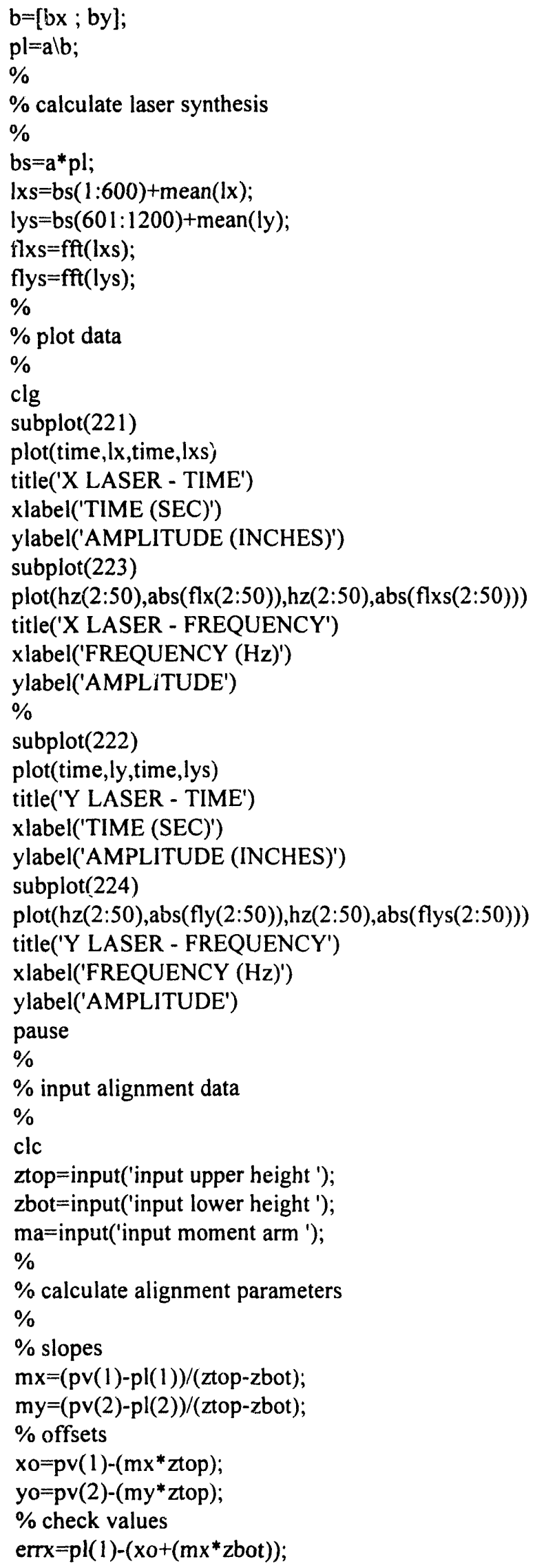




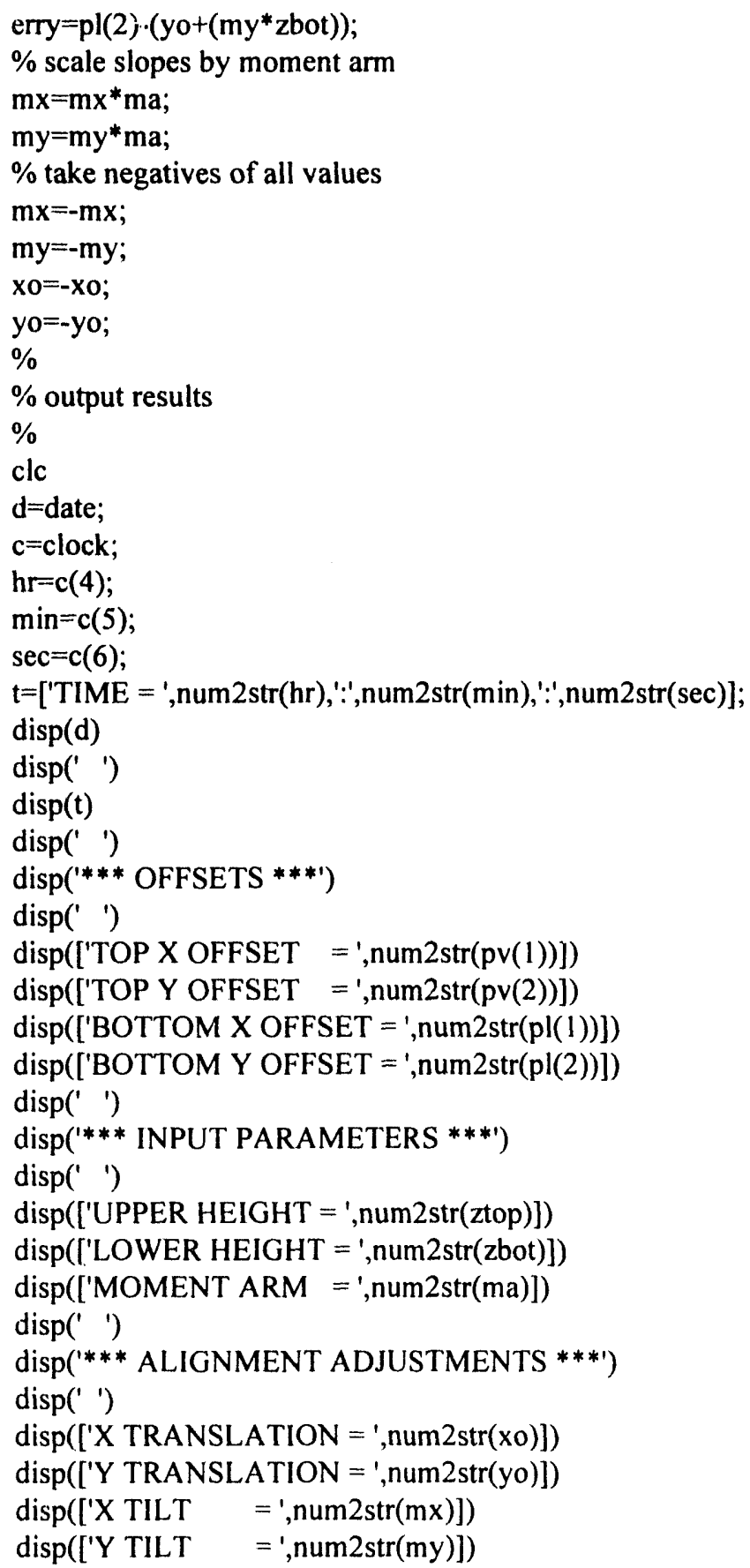


APPENDIX C

INTERPRETATION AND HARMONIC CURVE FIT OF VIDEO DATA 


\section{APPENDIX C \\ INTERPRETATION AND HARMONIC CURVE FIT OF VIDEO DATA}

The path traced out by the reference marker in the video data is an actual measurement of the orbit of one point on the reference axis. The orbit will be a perfect circle if the reference axis is not aligned with the machine spin axis. A single point results if the axes are aligned. Figure $\mathrm{C}-1$ shows the path or orbit traced out by the reference axis which is offset from the spin axis by $\left(X_{0}, Y_{0}\right)$ at the top measurement plane. The centers of the small circles represent the position of the reference axis at four angular values $\left(0^{\circ}, 90^{\circ}, 180^{\circ}\right.$, and $\left.270^{\circ}\right) .7$.e small line denotes the angular orientation of the body as measured between the reference marker and the angle marker. The large circle provides the path the reference axis makes during one revolution on the spin machine.

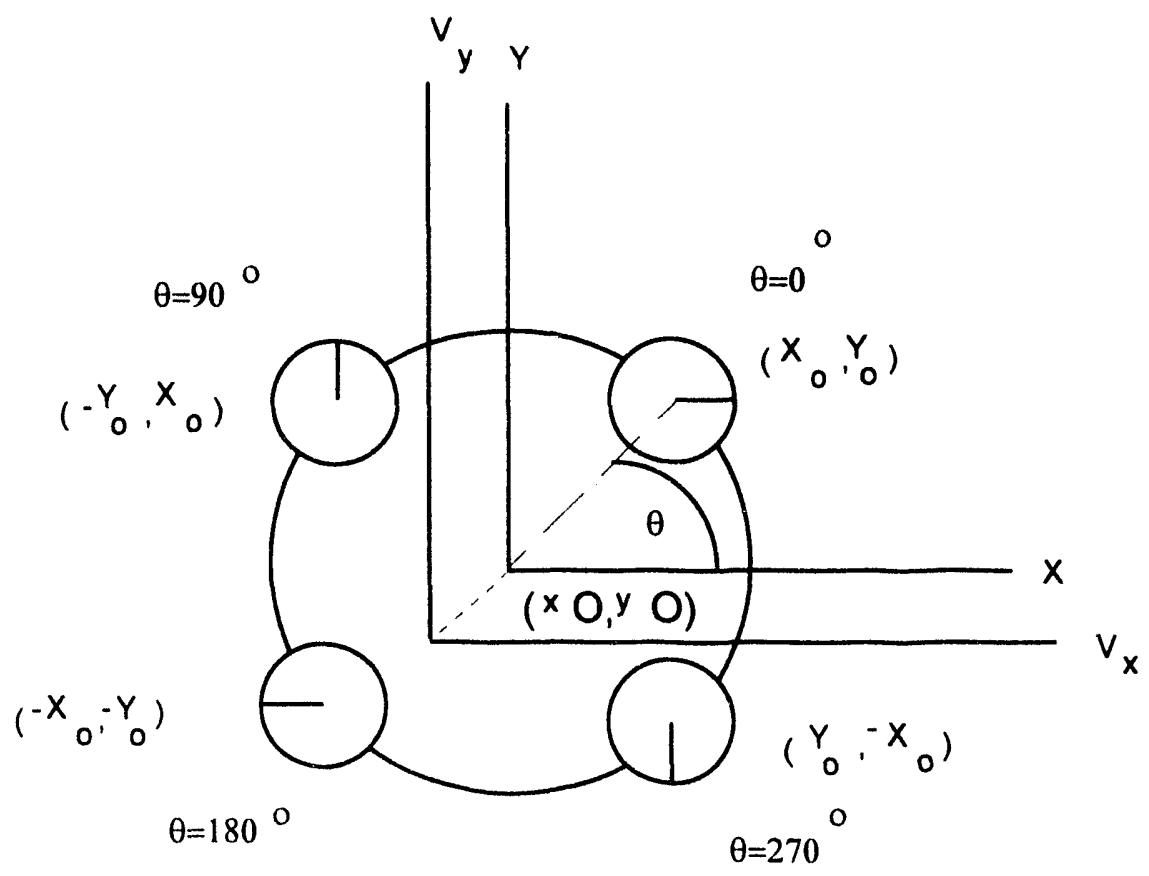

where $\quad V_{x}$ and $V_{y}$ are the fixed axes as defined by the video system,

$\mathrm{x}_{\mathrm{O}}$ and $\mathrm{y}_{\mathrm{O}}$ are the coordinates of the spin axis in the fixed axes, $X$ and $Y$ are the machine axes with origin at the spin axis,

$X_{0}$ and $Y_{0}$ are the offsets of the reference axis from the spin axis, and

$\theta$ is the angular orientation $m$ asured by the video system.

Figure C-1. Path Traced Out By Offset Reference Axis Marker

The mathematical relationship for the $\mathrm{x}$ and $\mathrm{y}$ values of the reference axis position as measured by the video camera take the following form:

$$
\begin{aligned}
& x={ }^{x} O+X_{0} \cos (\theta)-Y_{0} \sin (\theta) ; \text { and } \\
& y={ }^{y} O+X_{0} \sin (\theta)+Y_{0} \cos (\theta) .
\end{aligned}
$$


Equations (1) and (2) contain static offset terms ( ${ }^{x_{O}}$ and $\left.y_{O}\right)$ which allow the position of the spin axis to be determined as well as the reference axis offsets $\left(X_{0}\right.$ and $\left.Y_{0}\right)$. Equations (1) and (2) can be collected in the following matrix equations:

$$
\left\{\begin{array}{l}
x \\
y
\end{array}\right\}=\left[\begin{array}{cccc}
\cos (\theta) & -\sin (\theta) & 1 & 0 \\
\sin (\theta) & \cos (\theta) & 0 & 1
\end{array}\right]\left\{\begin{array}{c}
X_{0} \\
Y_{0} \\
x O \\
y_{O}
\end{array}\right\}
$$

However, data points $\mathrm{i}=1, \mathrm{n}$ are available and the following expanded set of equations results:

$$
\{B\}=\left\{\begin{array}{c}
x_{1} \\
x_{2} \\
\bullet \\
\bullet \\
\bullet \\
x_{n} \\
y_{1} \\
y_{2} \\
\bullet \\
\bullet \\
\bullet \\
y_{n}
\end{array}\right\}=\left[\begin{array}{cccc}
\cos \left(\theta_{1}\right) & -\sin \left(\theta_{1}\right) & 1 & 0 \\
\cos \left(\theta_{2}\right) & -\sin \left(\theta_{2}\right) & 1 & 0 \\
\bullet & \bullet & \bullet & \bullet \\
\bullet & \bullet & \bullet & \bullet \\
\bullet & \bullet & \bullet & \bullet \\
\cos \left(\theta_{n}\right) & -\sin \left(\theta_{n}\right) & 1 & 0 \\
\hdashline \sin \left(\theta_{1}\right) & \cos \left(\theta_{1}\right) & 0 & 1 \\
\sin \left(\theta_{2}\right) & \cos \left(\theta_{2}\right) & 0 & 1 \\
\bullet & \bullet & \bullet & \bullet \\
\bullet & \bullet & \bullet & \bullet \\
\bullet & \bullet & \bullet & \bullet \\
\sin \left(\theta_{n}\right) & \cos \left(\theta_{n}\right) & 0 & 1
\end{array}\right]\left\{\begin{array}{c}
X_{0} \\
Y_{0} \\
{ }^{\prime} O \\
{ } O
\end{array}\right\}=[A]\{P\}
$$

Six hundred data points are used in the implementation of this procedure as provided in the body of the text. Now the vector $\{\mathrm{P}\}$ can be solved for with a traditional least squares solution:

$$
\{P\}=\left[A^{T} A\right]^{-1}\left[A^{T}\right]\{B\}
$$

Now, the curve fit can be verified by synthesizing the data vector $\{B\}$ :

$$
\left\{{ }^{s} B\right\}=[A]\{P\} \text {. }
$$

The synthesized data and the original data can then be plotted together. 

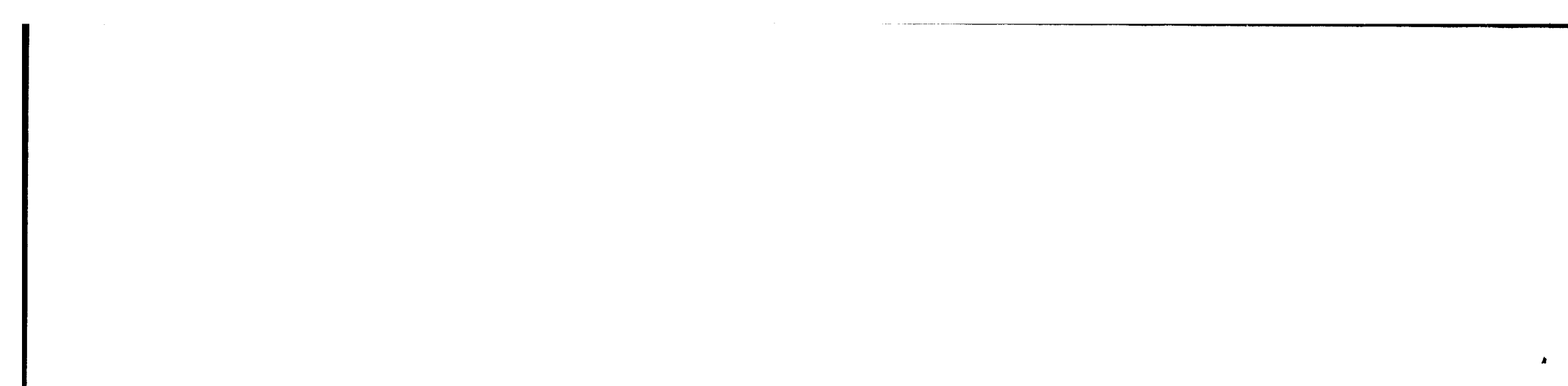

C-4

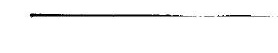


APPENDIX D

INTERPRETATION AND HARMONIC CURVE FIT OF LASER DATA

D-1 


\section{APPENDIX D \\ INTERPRETATION AND HARMONIC CURVE FIT OF LASER DATA}

\section{The Perfect Circle Simplification}

The information obtained from the laser triangulation devices is slightly more difficult to use and interpret than the video data. An important step in understanding and interpreting the laser data is gained by solving a simplified problem. The surface of the body being measured is assumed to be a perfect circle in this section. A more general definition of the surface is allowed in the next section. And finally, the third section of this appendix uses the derivations of the first two sections to describe the actual numerical interpretation of the laser data used in the matlab m-file CALC.M. Figure D-1 shows the geometry of the situation which will be described in this section. The goal is to describe mathematically the radial displacement of an offset circle.

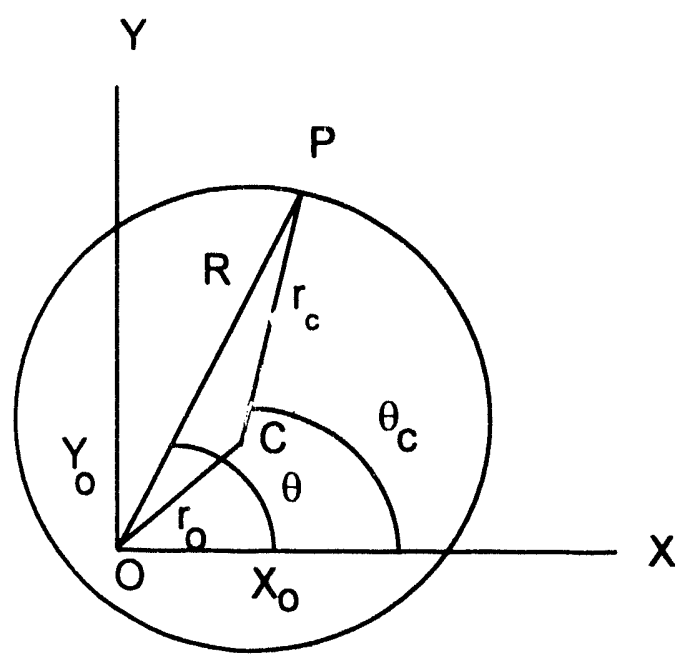

where $\mathrm{X}$ and $\mathrm{Y}$ are the machine coordinates with origin at the spin axis,

$R$ is the radial distance measured from the spin axis to the object surface (line OP), $\theta$ is the angular orientation measure by the video system (angle of OP w.r.t. X), $r_{0}$ is the distance from the origin (spin axis) to the center of the circle (line OC), $X_{0}$ and $Y_{0}$ are components of $r_{0}$ and are to be estimated and subsequently corrected, $r_{c}$ is the radius of the circle (line $C P$ ), and

$\theta_{c}$ is the angle of CP w.r.t. $X$.

\section{Figure D-1. The Perfect Offset Circle}

The governing equation for this situation is as follows:

$$
r_{c}^{2}=\left(x-X_{0}\right)^{2}+\left(y-Y_{0}\right)^{2} \text {. }
$$

The equation thus becomes upon expanding:

$$
x^{2}-2 x X_{0}+y^{2}-2 y Y_{0}=r_{c}^{2}-\left(X_{0}^{2}+Y_{0}^{2}\right) \text {. }
$$


Now converting to polar coordinates:

$$
\begin{aligned}
& x=R \cos (\theta) \Rightarrow x^{2}=R^{2} \cos ^{2}(\theta), \text { and } \\
& y=R \sin (\theta) \Rightarrow y^{2}=R^{2} \sin ^{2}(\theta) .
\end{aligned}
$$

Substituting (3) and (4) into (2) provides the following:

$$
R^{2}-\left[2\left(X_{0} \cos (\theta)+Y_{0} \sin (\theta)\right)\right] R+\left[\left(X_{0}^{2}+Y_{0}^{2}\right)-r_{c}^{2}\right]=R^{2}+b R+c=0
$$

After applying the following identity:

$$
\cos ^{2}(\theta)+\sin ^{2}(\theta)=1.0
$$

It should be noted that values for radial displacement $(R)$ and angular orientation $(\theta)$ are to be measured and values for the offset $\left(X_{0}\right.$ and $\left.Y_{0}\right)$ are to be calculated. Now solve for $R$ by the quadratic formula:

$$
R=\frac{-b \pm \sqrt{b^{2}-4 c}}{2}
$$

With the following defined using trig power relations:

$$
b^{2}-4 c=4 r_{c}^{2}\left\{1-\left[\left(\frac{X_{0}^{2}+Y_{0}^{2}}{2 r_{c}^{2}}\right)-\left(\frac{X_{0} Y_{0}}{r_{c}^{2}}\right) \sin (2 \theta)-\left(\frac{X_{0}^{2}-Y_{0}^{2}}{2 r_{c}^{2}}\right) \cos (2 \theta)\right]\right\} \text {. }
$$

Now the following results using a binomial expansion, trig identities, and renaming the coefficients:

$$
\begin{aligned}
& \sqrt{b^{2}-4 c}=2\left\{r_{c}+C_{0}+C_{1} \sin (2 \theta)+C_{2} \cos (2 \theta)+C_{3} \sin (4 \theta)+C_{4} \cos (4 \theta)+\ldots\right\} ; \\
& \text { where } C_{i} \text { are functions of } r_{c} x_{0} \text {, and } Y_{0} .
\end{aligned}
$$

After choosing a plus sign for a positive radius, the following measured distance results:

$$
R=\left(r_{c}+C_{0}\right)+X_{0} \cos (\theta)+Y_{0} \sin (\theta)+C_{1} \cos (2 \theta)+C_{2} \sin (2 \theta)+\ldots
$$

The important results of the perfect offset circle derivation are the following:

1. The desired offsets are coefficients of the first order trig functions,

2. No approximations were used,

3. Higher order response will appear as even power harmonics of the rotation rate, and

4. The measured radius reduces to a constant when the offsets are zero.

\section{Results for a More General Surface}

The derivation of the results for a more general surface will begin by assuming that there is a best fitting circle such that when the object is aligned the coefficients of the first order trig functions disappear. The center of this best fitting circle will be the reference axis to which alignment is made. Figure D-2 pictures such a situation: 


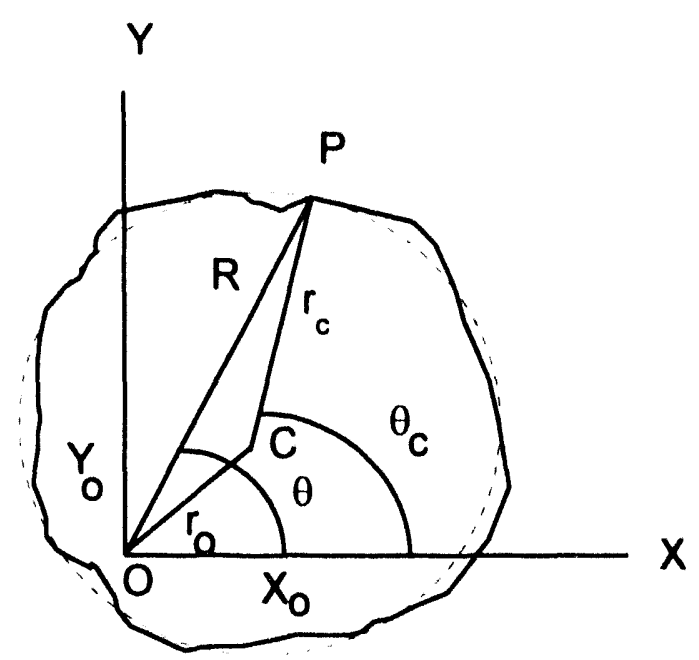

where $\mathrm{X}$ and $\mathrm{Y}$ are the machine coordinates with origin at the spin axis,

$R$ is the radial distance measured from the spin axis to the object surface (line OP), $\theta$ is the angular orientation measured by the video system (angle of OP w.r.t. $X$ ), $r_{0}$ is the distance from the origin (spin axis) to the reference axis (line $O C$ ),

$X_{0}$ and $Y_{0}$ are components of $r_{0}$ and are to be estimated and subsequently corrected, $r_{c}$ is the distance from the reference axis to the surface (line $C P$ ), and $\theta_{c}$ is the angle of CP w.r.t. $X$ and assumed to be equal to $\theta$ for small $r_{0}$.

\section{Figure D-2. A General Offset Shape With an Associated Perfect Circle}

The resulting polar description of the surface as measured from the reference axis is modeled as follows:

$$
\begin{gathered}
r_{c}=P_{0}+P_{1} \cos \left(2 \theta_{c}\right)+P_{2} \sin \left(2 \theta_{c}\right)+P_{3} \cos \left(3 \theta_{c}\right)+P_{4} \sin \left(3 \theta_{c}\right)+\ldots \\
+P_{2 m-3} \cos \left(m \theta_{c}\right)+P_{2 m-2} \sin \left(m \theta_{c}\right) .
\end{gathered}
$$

A small $r_{0}$ is assumed $\left(q_{c} \approx q\right)$ and the following statement can also be made:

$$
\begin{gathered}
r_{c} \approx P_{0}+P_{1} \cos (2 \theta)+P_{2} \sin (2 \theta)+P_{3} \cos (3 \theta)+P_{4} \sin (3 \theta)+\ldots \\
+P_{2 m-3} \cos (m \theta)+P_{2 m-2} \sin (m \theta) .
\end{gathered}
$$

Now if $\mathrm{R}$ and $\mathrm{r}_{\mathrm{c}}$ are converted into polar coordinates then the following components result;

$$
\begin{aligned}
& x_{c}=r_{c} \cos (\theta), \\
& y_{c}=r_{c} \sin (\theta), \\
& x=R \cos (\theta), \text { and } \\
& y=R \sin (\theta) .
\end{aligned}
$$

Since we are actually performing vector sums the following relationships between the components hold:

$$
\begin{aligned}
& x=X_{0}+x_{c}, \text { and } \\
& y=Y_{0}+y_{c} .
\end{aligned}
$$


And using equations (15) - (18), the following relationship results for the magnitude of $R$ :

$$
R=\sqrt{x^{2}+y^{2}}=\sqrt{\left(X_{0}+x_{c}\right)^{2}+\left(Y_{0}+y_{c}\right)^{2}} .
$$

Using (13) and (14) and the small offset approximation, which allows us to neglect second order terms in $X_{0}$ and $Y_{0}$, equation (19) can be rewritten:

$$
R \approx \sqrt{\left(x_{c}^{2}+y_{c}^{2}\right)+2\left(x_{c} X_{0}+y_{c} Y_{0}\right)}=r_{c} \sqrt{1+\frac{2}{r_{c}^{2}}\left(x_{c} X_{0}+y_{c} Y_{0}\right)}
$$

Now the binomial theorem expansion and the small offset approximation coupled with (13) and (14) allows the following approximation of $R$ :

$$
R \approx r_{c}\left[1+\frac{1}{r_{c}^{2}}\left(x_{c} X_{0}+y_{c} Y_{0}\right)\right]=r_{c}+X_{0} \frac{x_{c}}{r_{c}}+Y_{0} \frac{y_{c}}{r_{c}}=r_{c}+X_{0} \cos (\theta)+Y_{0} \sin (\theta) .
$$

Substituting (12) into (21) results in the following:

$$
\begin{gathered}
R \approx X_{0} \cos (\theta)+Y_{0} \sin (\theta)+P_{0}+P_{1} \cos (2 \theta)+P_{2} \sin (2 \theta)+\ldots \\
+P_{2 m-3} \cos (m \theta)+P_{2 m-2} \sin (m \theta)
\end{gathered}
$$

And finally, equation (22) can be placed in matrix form for several measurements at various timesteps:

$$
\left\{\begin{array}{l}
R_{0} \\
R_{1} \\
R_{2} \\
\bullet \\
\bullet \\
\bullet \\
R_{n}
\end{array}\right\}=\left[\begin{array}{cccccccccc}
1 & c \theta_{0} & s \theta_{0} & c 2 \theta_{0} & s 2 \theta_{0} & \bullet & \bullet & \bullet & c m \theta_{0} & s m \theta_{0} \\
1 & c \theta_{1} & s \theta_{1} & c 2 \theta_{1} & s 2 \theta_{1} & \bullet & \bullet & \bullet & c m \theta_{1} & s m \theta_{1} \\
1 & c \theta_{2} & s \theta_{2} & c 2 \theta_{2} & s 2 \theta_{2} & \bullet & \bullet & \bullet & c m \theta_{2} & s m \theta_{2} \\
\bullet & \bullet & \bullet & \bullet & \bullet & \bullet & & \bullet & \bullet \\
\bullet & \bullet & \bullet & \bullet & \bullet & \bullet & \bullet & \bullet \\
\bullet & \bullet & \bullet & \bullet & \bullet & & \bullet & \bullet & \bullet \\
1 & c \theta_{n} & \mathrm{~s} \theta_{n} & c 2 \theta_{n} & s 2 \theta_{n} & \bullet & \bullet & \bullet & c m \theta_{n} & s m \theta_{n}
\end{array}\right]\left\{\begin{array}{c}
P_{0} \\
X_{0} \\
Y_{0} \\
P_{1} \\
P_{2} \\
P_{3} \\
P_{4} \\
\bullet \\
\bullet \\
P_{2 m-3} \\
P_{2 m-2}
\end{array}\right\}
$$


Hence, this section has developed a description of the radial measurements as would be seen from a body of general shape in which the reference axis is offset from the spin axis. Two important points must be remembered: a small offset was assumed and the reference axis was defined as the center of a best fit circle. Placing the center of this circle on the spin axis of the machine eliminated the coefficients of the first order trig functions of the general shape description. And finally, a matrix formulation of the results was given. The next section will provide the actual implementation of these concepts as described in the body of this report.

\section{Actual Implementation of Laser Data Curve Fit}

The actual configuration of the laser displacement sensors includes one sensor viewing the radial displacement of the object surface from the $X$ direction $\left(0^{\circ}\right)$ and another viewing the surface from the $Y$ direction $\left(90^{\circ}\right)$. When both are at the same height, these devises are measuring the same shape and hence the same harmonic coefficients of equation (22) and (23). The one exception is the offset term $P_{0}$ which is unique for each sensor head. This allows the sensors to be at slightly different distances from the surface without affecting data quality. These offset parameters will be denoted by ${ }^{0} \mathrm{O}$ and ${ }^{90} \mathrm{O}$ respectively for the $X$ and $Y$ sensors. Additionally, experience has shown that seven harmonics can usually be used to successfully describe the major features of the shape of the objects tested. Therefore, the following parameter vector is defined:

$$
\{\mathrm{P}\}^{\mathrm{T}}=\left\{\begin{array}{llllllllllllllll}
{ }^{0} \mathrm{O} & { }^{90} \mathrm{O} & \mathrm{X}_{0} & \mathrm{Y}_{0} & \mathrm{P}_{1} & \mathrm{P}_{2} & \mathrm{P}_{3} & \mathrm{P}_{4} & \mathrm{P}_{5} & \mathrm{P}_{6} & \mathrm{P}_{7} & \mathrm{P}_{8} & \mathrm{P}_{9} & \mathrm{P}_{10} & \mathrm{P}_{11} & \mathrm{P}_{12}
\end{array}\right\} .
$$

A matrix similar to that defined in equation (23) can then easily be constructed for the $0^{\circ}$ laser:

$\left[{ }^{0} A\right]=\left[\begin{array}{ccccccccccccccccc}1 & 0 & c \theta_{0} & \mathrm{~s} \theta_{0} & \mathrm{c} 2 \theta_{0} & \mathrm{~s} 2 \theta_{0} & \mathrm{c} 3 \theta_{0} & \mathrm{~s} 3 \theta_{0} & \mathrm{c} 4 \theta_{0} & \mathrm{~s} 4 \theta_{0} & \mathrm{c} 5 \theta_{0} & \mathrm{~s} 5 \theta_{0} & \mathrm{c} 6 \theta_{0} & \mathrm{~s} 6 \theta_{0} & \mathrm{c} 7 \theta_{0} & \mathrm{~s} 7 \theta_{0} \\ 1 & 0 & \mathrm{c} \theta_{1} & \mathrm{~s} \theta_{1} & \mathrm{c} 2 \theta_{1} & \mathrm{~s} 2 \theta_{1} & \mathrm{c} 3 \theta_{1} & \mathrm{~s} 3 \theta_{1} & \mathrm{c} 4 \theta_{1} & \mathrm{~s} 4 \theta_{1} & \mathrm{c} 5 \theta_{1} & \mathrm{~s} 5 \theta_{1} & \mathrm{c} 6 \theta_{1} & \mathrm{~s} 6 \theta_{1} & \mathrm{c} 7 \theta_{1} & \mathrm{~s} 7 \theta_{1} \\ 1 & 0 & \mathrm{c} \theta_{2} & \mathrm{~s} \theta_{2} & \mathrm{c} 2 \theta_{2} & \mathrm{~s} 2 \theta_{2} & \mathrm{c} 3 \theta_{2} & \mathrm{~s} 3 \theta_{2} & \mathrm{c} 4 \theta_{2} & \mathrm{~s} 4 \theta_{2} & \mathrm{c} 5 \theta_{2} & \mathrm{~s} 5 \theta_{2} & \mathrm{c} 6 \theta_{2} & \mathrm{~s} 6 \theta_{2} & \mathrm{c} 7 \theta_{2} & \mathrm{~s} 7 \theta_{2} \\ \bullet & \bullet & \bullet & \bullet & \bullet & \bullet & \bullet & \bullet & \bullet & \bullet & \bullet & \bullet & \bullet & \bullet & \bullet & \bullet \\ \bullet & \bullet & \bullet & \bullet & \bullet & \bullet & \bullet & \bullet & \bullet & \bullet & \bullet & \bullet & \bullet & \bullet & \bullet & \bullet \\ \bullet & \bullet & \bullet & \bullet & \bullet & \bullet & \bullet & \bullet & \bullet & \bullet & \bullet & \bullet & \bullet & \bullet & \bullet & \bullet \\ 1 & 0 & c \theta_{n} & \mathrm{~s} \theta_{\mathrm{n}} & \mathrm{c} 2 \theta_{\mathrm{n}} & \mathrm{s} 2 \theta_{\mathrm{n}} & \mathrm{c} 3 \theta_{\mathrm{n}} & \mathrm{s} 3 \theta_{\mathrm{n}} & \mathrm{c} 4 \theta_{\mathrm{n}} & \mathrm{s} 4 \theta_{\mathrm{n}} & \mathrm{c} 5 \theta_{\mathrm{n}} & \mathrm{s} 5 \theta_{\mathrm{n}} & \mathrm{c} 6 \theta_{\mathrm{n}} & \mathrm{s} 6 \theta_{\mathrm{n}} & \mathrm{c} 7 \theta_{\mathrm{n}} & \mathrm{s} 7 \theta_{\mathrm{n}}\end{array}\right]$

(D-25)

The extra column of zeros has been added since the data from the $\mathrm{X}$ direction laser is not dependent upon the offset of the $Y$ direction laser. Also, in the current implementation, 600 timesteps are used $(n=600)$. The $Y$ direction laser is measuring the position of the surface at an angular position advanced $90^{\circ}$ from the $\mathrm{X}$ laser. Therefore, all transcendental functions must be advanced by $90^{\circ}$ as shown below:

$$
\begin{gathered}
\cos (90+\theta)=-\sin (\theta) ; \\
\sin (90+\theta)=\cos (\theta) ; \\
\cos [2(90+\theta)]=\cos (180+2 \theta)=-\cos (2 \theta) ; \\
\sin [2(90+\theta)]=\sin (180+2 \theta)=-\sin (2 \theta) ; \\
\cos [3(90+\theta)]=\cos (270+3 \theta)=\sin (3 \theta) ; \\
\sin [3(90+\theta)]=\sin (270+3 \theta)=-\cos (3 \theta) ; \\
\cos [4(90+\theta)]=\cos (360+4 \theta)=\cos (4 \theta) ;
\end{gathered}
$$




$$
\begin{gathered}
\sin [4(90+\theta)]=\sin (360+4 \theta)=\sin (4 \theta) \\
\cos [5(90+\theta)]=\cos (90+5 \theta)=-\sin (5 \theta) \\
\sin [5(90+\theta)]=\sin (90+5 \theta)=\cos (5 \theta) \\
\cos [6(90+\theta)]=\cos (180+6 \theta)=-\cos (6 \theta) \\
\sin [6(90+\theta)]=\sin (180+6 \theta)=-\sin (6 \theta) \\
\cos [7(90+\theta)]=\cos (270+7 \theta)=\sin (7 \theta) ; \text { and } \\
\sin [7(90+\theta)]=\sin (270+7 \theta)=-\cos (7 \theta)
\end{gathered}
$$

Substituting equations (26) - (39) into the matrix defined in (25) results in the following matrix for the Y laser:

$\left[{ }^{90} A\right]=\left[\begin{array}{cccccccccccccccc}0 & 1 & -\mathrm{s} \theta_{0} & \mathrm{c} \theta_{0} & -\mathrm{c} 2 \theta_{0} & -\mathrm{s} 2 \theta_{0} & \mathrm{~s} 3 \theta_{0} & -\mathrm{c} 3 \theta_{0} & \mathrm{c} 4 \theta_{0} & \mathrm{~s} 4 \theta_{0} & -\mathrm{s} 5 \theta_{0} & \mathrm{c} 5 \theta_{0} & -\mathrm{c} 6 \theta_{0} & -\mathrm{s} 6 \theta_{0} & \mathrm{~s} 7 \theta_{0} & -\mathrm{c} 7 \theta_{0} \\ 0 & 1 & -\mathrm{s} \theta_{1} & \mathrm{c} \theta_{1} & -\mathrm{c} 2 \theta_{1} & -\mathrm{s} 2 \theta_{1} & \mathrm{~s} 3 \theta_{1} & -\mathrm{c} 3 \theta_{1} & \mathrm{c} 4 \theta_{1} & \mathrm{~s} 4 \theta_{1} & -\mathrm{s} 5 \theta_{1} & \mathrm{c} 5 \theta_{1} & -\mathrm{c} 6 \theta_{1} & -\mathrm{s} 6 \theta_{1} & \mathrm{~s} 7 \theta_{1} & -\mathrm{c} 7 \theta_{1} \\ 0 & 1 & -\mathrm{s} \theta_{2} & \mathrm{c} \theta_{2} & -\mathrm{c} 2 \theta_{2} & -\mathrm{s} 2 \theta_{2} & \mathrm{~s} 3 \theta_{2} & -\mathrm{c} 3 \theta_{2} & \mathrm{c} 4 \theta_{2} & \mathrm{~s} 4 \theta_{2} & -\mathrm{s} 5 \theta_{2} & \mathrm{c} 5 \theta_{2} & -\mathrm{c} 6 \theta_{2} & -\mathrm{s} 6 \theta_{2} & \mathrm{~s} 7 \theta_{2} & -\mathrm{c} 7 \theta_{2} \\ \bullet & \bullet & \bullet & \bullet & \bullet & \bullet & \bullet & \bullet & \bullet & \bullet & \bullet & \bullet & \bullet & \bullet & \bullet & \bullet \\ \bullet & \bullet & \bullet & \bullet & \bullet & \bullet & \bullet & \bullet & \bullet & \bullet & \bullet & \bullet & \bullet & \bullet & \bullet & \bullet \\ \bullet & \bullet & \bullet & \bullet & \bullet & \bullet & \bullet & \bullet & \bullet & \bullet & \bullet & \bullet & \bullet & \bullet & \bullet & \bullet \\ 0 & 1 & -\mathrm{s} \theta_{\mathrm{n}} & \mathrm{c} \theta_{\mathrm{n}} & -\mathrm{c} 2 \theta_{\mathrm{n}} & -\mathrm{s} 2 \theta_{\mathrm{n}} & \mathrm{s} 3 \theta_{\mathrm{n}} & -\mathrm{c} 3 \theta_{\mathrm{n}} & \mathrm{c} 4 \theta_{\mathrm{n}} & \mathrm{s} 4 \theta_{\mathrm{n}} & -\mathrm{s} 5 \theta_{\mathrm{n}} & \mathrm{c} 5 \theta_{\mathrm{n}} & -\mathrm{c} 6 \theta_{\mathrm{n}} & -\mathrm{s} 6 \theta_{\mathrm{n}} & \mathrm{s} 7 \theta_{\mathrm{n}} & -\mathrm{c} 7 \theta_{\mathrm{n}}\end{array}\right]$

The final problem to be solved begins by collecting the 600 measured data point from the $X$ laser and the 600 measured points from the $Y$ laser into a single vector, $\{R\}$. Matrix equations (25) and (40) are collected into a single $1200 \times 16$ matrix, [A]. The resulting matrix equation is shown below:

$$
\{R\}=\left\{\begin{array}{c}
{ }^{0} R \\
{ }_{90} \cdots \\
{ }^{90} R
\end{array}\right\}=\left[\begin{array}{c}
{ }^{0} A \\
{ }_{90} A
\end{array}\right]\{P\}=[A]\{P\} .
$$

A least squares estimate of the parameter vector, $\{\mathrm{P}\}$, can then be calculated as shown below:

$$
\{P\}=\left[A^{T} A\right]^{-1}[A]^{T}\{R\} .
$$

The second and third entries in the parameter vector are therefore the offsets of the reference axis (as defined by the best fit circle) from the spin axis of the machine. The angle theta is known only by assuming small offsets as described in the previous section. A further complication is that the angular meası rements occur at the top plane. However, the object can be easily placed such that the appropriate small offset assumptions hold. As a final note, a resynthesized data record, $\left\{{ }^{\mathbf{S}} \mathrm{R}\right\}$, can be calculated and compared with the original data, $\{R\}$, for a verification of the curve fit:

$$
\left\{{ }^{s} \mathrm{R}\right\}=[\mathrm{A}]\{\mathrm{P}\} .
$$

It is also useful to compare synthesized and original data in the frequency domain. A peak for each of the seven harmonics should be seen. Also, the first peak will shrink as the object is aligned. Upon alignment, the center of the best fitting circle through the area is on the reference axis of the body. Therefore, the primary function of these calculations has been to define that reference axis. 
APPENDIX E

ALIGNMENT CALCULATION 


\section{APPENDIX E}

\section{ALIGNMENT CALCULATION}

The calculation of the adjustments needed for alignment assumes that the top and bottom offsets $\left(v_{X}\right.$ and $\left.I_{x}\right)$ are available as well as the heights of the top and bottom measurements $\left(z_{\text {top }}\right.$ and $\left.z_{\text {bot }}\right)$ and the moment arm for the tilt adjustments $\left(m_{a}\right)$. The program calculates the line (this is actually the $X$ coordinate of the reference axis) which passes through the top and bottom reference points. The slope and intercept of this line then provides the necessary adjustments. It should be noted that the $X$ and $Y$ coordinates will be treated separately and hence there will be two lines. Figure $E-1$ contains the geometry used for the $\mathrm{X}$ direction calculations.

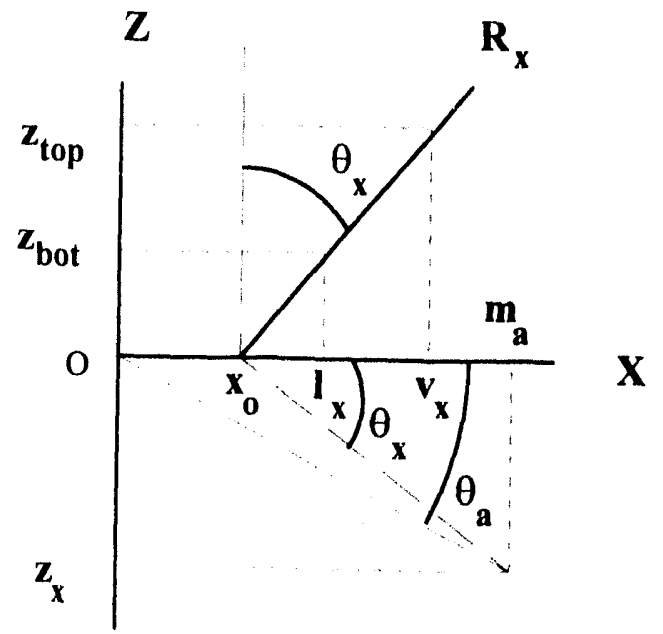

where $\quad v_{x}$ is the calculated offset of the top reference point,

$I_{X}$ is the calculated offset of the bottom reference point,

$z_{\text {top }}$ is the height of the top measurement point,

$Z_{\text {bot }}$ is the height of the bottom measurement point,

$\mathrm{O}$ is the origin at the center of the tilt/translation ball,

$\mathrm{m}_{\mathrm{a}}$ is the moment arm at which the tilt adjustments will be made,

$R_{X}$ is the $x$ component of the reference line,

$\theta_{x}$ is the angle the reference line makes with the vertical axis,

$x_{0}$ is the intercept of the reference line,

$\theta_{\mathrm{a}}$ is the angle the tilt adjustments will produce, and

$\mathrm{z}_{\mathrm{X}}$ is the actual tilt adjustment.

Figure E-1. Geometry Used to Calculate Adjustments to Remove X Offsets

The slope of the reference line $R_{X}$ is calculated as follows:

$$
m_{x}=\frac{v_{x}-l_{x}}{z_{\text {top }}-z_{b o t}}
$$

And the intercept of the reference line $R_{X}$ is calculated as follows:

$$
x_{0}=v_{x}-m_{x}\left(z_{t o p}\right)=l_{x}-m_{x}\left(z_{b o t}\right)
$$


Now, the angle $\theta_{\mathbf{X}}$ is defined as follows:

$$
\tan \left(\theta_{x}\right)=\frac{v_{x}-x_{0}}{z_{\text {top }}}=m_{x}
$$

However, since $\theta_{\mathbf{X}}$ is a very small angle the following approximation is valid:

$$
\theta_{x} \cong m_{x}
$$

Now lets explore the relationship between $\theta_{\mathbf{a}}$ and $\theta_{\mathbf{X}}$ assuming that both are small angles:

$$
\theta_{a} \cong \tan \left(\theta_{a}\right)=\frac{z_{x}}{m_{a}}=\left(\frac{m_{a}-x_{0}}{m_{a}}\right) \frac{z_{x}}{m_{a}-x_{0}}=\left(1-\frac{x_{0}}{m_{a}}\right) \tan \left(\theta_{x}\right) \cong\left(1-\frac{x_{0}}{m_{a}}\right) \theta_{x} .
$$

The difference between $\theta_{\mathrm{a}}$ and $\theta_{\mathrm{x}}$ is typically ignored, however this would be one possible upgrade to the procedure. The adjustment needed to drive $x_{0}$ to zero is a translation in the $X$ direction by $-x_{0}$. The vertical adjustment $\left(\mathrm{z}_{\mathrm{X}}\right)$ needed to correct the tilt is then the following:

$$
z_{x}=\tan \left(-\theta_{a}\right) m_{a}=-\tan \left(\theta_{a}\right) m_{a} \cong-\tan \left(\theta_{x}\right) m_{a} \cong-m_{x} m_{a}
$$

The $\mathrm{Y}$ coordinate calculations proceed similarly. Figure E-2 contains the geometry used for the $Y$ direction calculations. It should be noted that the tilt angle $\theta_{y}$ as shown is actually measured in a lefthanded coordinate system. The slope of the reference line $R_{y}$ is calculated as follows:

$$
m_{y}=\frac{v_{y}-l_{y}}{z_{t o p}-z_{b o t}}
$$

And the intercept of the reference line $\mathrm{R}_{\mathrm{y}}$ is calculated as follows:

$$
y_{0}=v_{y}-m_{y}\left(z_{\text {top }}\right)=l_{y}-m_{y}\left(z_{b o t}\right)
$$

Now, the angle $\theta_{\mathrm{y}}$ is defined as follows:

$$
\tan \left(\theta_{y}\right)=\frac{v_{y}-y_{0}}{z_{\text {top }}}=m_{y}
$$

However, since $\theta_{\mathrm{y}}$ is a very small angle the following approximation holds:

$$
\theta_{y} \cong m_{y} .
$$

As with the $X$ coordinate the relationship between the small angles of $\theta_{a}$ and $\theta_{y}$ is described as follows:

$$
\theta_{a} \cong \tan \left(\theta_{a}\right)=\frac{z_{y}}{m_{a}}=\left(\frac{m_{a}-y_{0}}{m_{a}}\right) \frac{z_{y}}{m_{a}-y_{0}}=\left(1-\frac{y_{0}}{m_{a}}\right) \tan \left(\theta_{y}\right) \cong\left(1-\frac{y_{0}}{m_{a}}\right) \theta_{y} \text {. }
$$

The difference between $\theta_{a}$ and $\theta_{y}$ is also typically ignored. The adjustment needed to drive $y_{0}$ to zero is a translation in the $Y$ direction by $-y_{0}$. The vertical adjustment $\left(z_{y}\right)$ needed to correct the tilt is then the following: 
$z_{y}=\tan \left(-\theta_{a}\right) m_{a}=-\tan \left(\theta_{a}\right) m_{a} \cong-\tan \left(\theta_{y}\right) m_{a} \cong-m_{y} m_{a}$

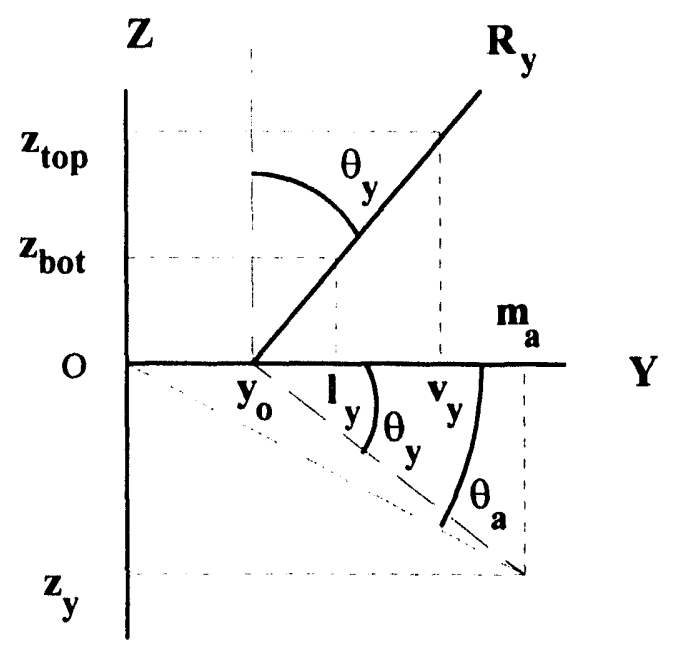

where $\quad v_{y}$ is the calculated offset of the top reference point,

$\mathrm{l}_{\mathrm{y}}$ is the calculated offset of the bottom reference point,

$z_{\text {top }}$ is the height of the top measurement point,

$z_{\text {bot }}$ is the height of the bottom measurement point,

$O$ is the origin at the center of the tilt/translation ball,

$\mathrm{m}_{\mathrm{a}}$ is the moment arm at which the tilt adjustments will be made,

Ry is the $y$ coordinate of the reference line,

$\theta_{y}$ is the angle the reference line makes with the vertical axis,

$y_{0}$ is the intercept of the reference line,

$\theta \mathrm{a}$ is the angle the tilt adjustments will produce, and

$z_{y}$ is the actual tilt adjustment.

Figure E-2. Geometry Used to Calculate Adjustments to Remove Y Offsets 


\section{APPENDIX F}

ALIGNMENT CALCULATION CORRECTED FOR CONICAL OBJECTS 


\section{APPENDIX F}

\section{ALIGNMENT CALCULATION CORRECTED FOR CONICAL OBJECTS}

Previous sections of this report have described techniques to determine the offsets of an object using a defined reference axis at a specific height and the calculation of the subsequent adjustments needed to remove these offsets. This entire approach works on the premise that there are no bias errors in the offsets. This premise holds very well for cylindrical objects, however the test objects are quite often conical bodies Unfortunately, the center of a tilted cross-section of a perfect cone is a ellipse with the center of the ellipse not on the axis of symmetry of the cone [9]. Therefore, a bias error is to be expected for the typical object of interest. It has been found in practice that these errors are not defeating and are handled quite well by iteration (as the offsets decrease as the object becomes more aligned with the machine spin axis).

The thrust of this appendix is to mathematically describe these bias errors and develop an alternate technique to calculate the necessary adjustments which takes these errors into account. The expected result is then a procedure which requires fewer iterations and therefore less time. The approach will begin by fully describing the relationship between the object geometry (which is assumed to be of a conical nature) and the fixed measurements. Then an idealized model of the object geometry will be used to estimate the measurements the sensors will see. After the bias terms are identified, a method for calculating the desired tilt angles and offsets will be developed.

\section{Derivation of Transformation Between Table and Body-Fixed Coordinates}

The first step in the derivation of an upgraded alignment calculation is the determination of a complete transformation from the fixed machine coordinates to the coordinates of the body. This requires the definition of three translations and three rotations and the associated seven coordinate systems. The machine coordinate system (X, Y, and Z) was defined using Figure 4 in the main report. All measurements and adjustments will be made in the machine coordinate system. The body coordinate system $\left(X_{b}, Y_{b}\right.$, and $\left.Z_{b}\right)$ will be used to define the shape of the test object.

The first transformation is a translation (denoted by the constant $\gamma$ ) along the third machine axis $(Z)$. This models the height of the body above the origin of the fixed coordinate system which was defined as the center of the spherical bearing of the Space Electronics tilt-translation table. The new coordinate system $\left(\mathrm{X}_{1}, \mathrm{Y}_{1}, \mathrm{Z}_{1}\right)$ is defined as follows:

$$
\begin{aligned}
& X_{1}=X, \\
& Y_{1}=Y, \text { and } \\
& Z_{1}=Z-\gamma .
\end{aligned}
$$

Equations (4)-(6) result if equations (1)-(3) are inverted:

$$
\begin{aligned}
& X=X_{1}, \\
& Y=Y_{1}, \text { and } \\
& Z=Z_{1}+\gamma .
\end{aligned}
$$


Figure F-1 depicts this transformation:

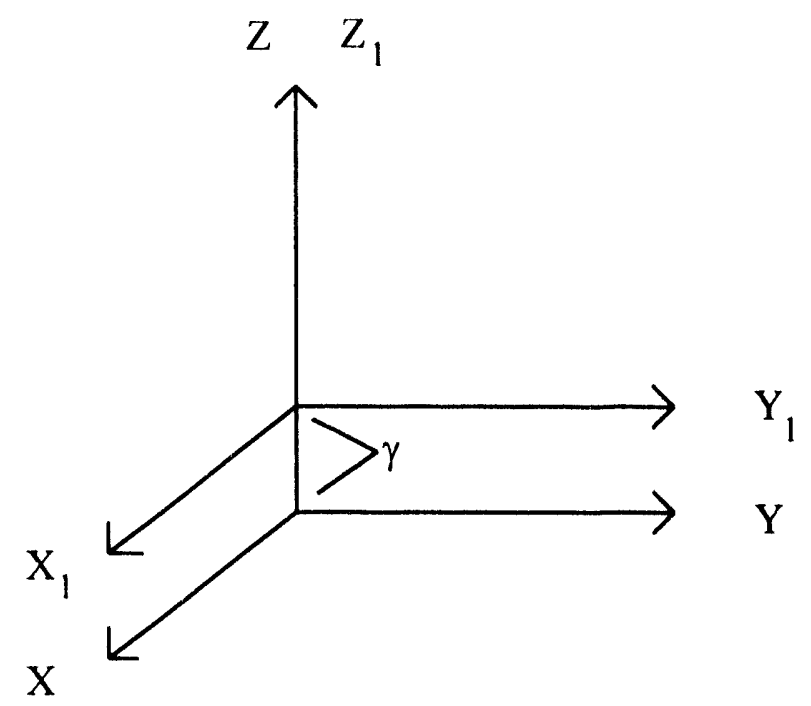

Figure F-1. First Transformation - Translation Along Z

The second transformation is a rotation about the $Z_{1}$ axis (denoted by the angle $\theta$ ) which models the spin of the machine. Figure F-2 depicts this situation as if the viewer is looking straight down the $Z_{1}$ (or $Z_{2}$ ) axis which is represented by the semi-shaded circle.

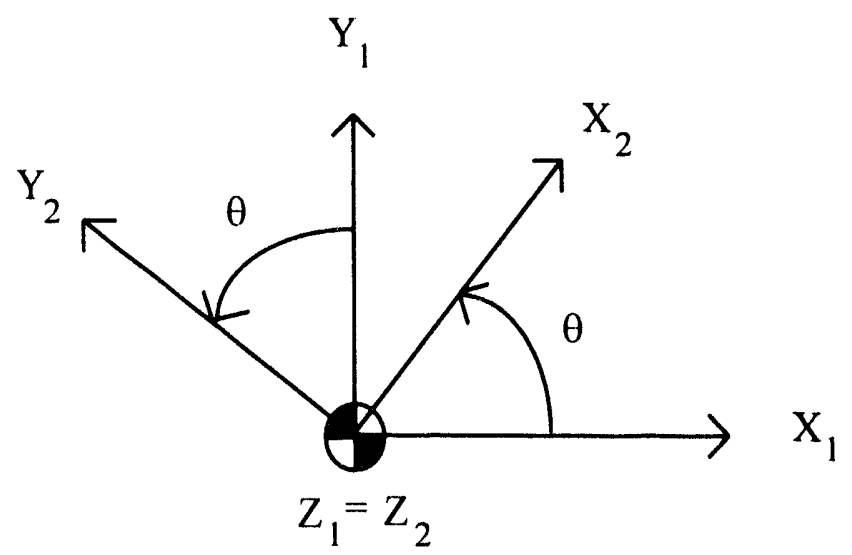

Figure F-2. Second Transformation - Rotation About $\mathrm{Z}_{1}$

The axes $X_{2}, Y_{2}$, and $Z_{2}$ are therefore defined as follows:

$$
\begin{gathered}
X_{2}=\cos (\theta) X_{1}+\sin (\theta) Y_{1}, \\
Y_{2}=-\sin (\theta) X_{1}+\cos (\theta) Y_{1} \text {, and } \\
Z_{2}=Z_{1} .
\end{gathered}
$$


Equations (10)-(12) result from inverting (7)-(9):

$$
\begin{gathered}
X_{1}=\cos (\theta) X_{2}-\sin (\theta) Y_{2}, \\
Y_{1}=\sin (\theta) X_{2}+\cos (\theta) Y_{2}, \text { and } \\
Z_{1}=Z_{2} .
\end{gathered}
$$

Now substituting equations (10)-(12) into (4)-(6) allows a description of the original coordinate system:

$$
\begin{aligned}
X= & \cos (\theta) X_{2}-\sin (\theta) Y_{2}, \\
Y= & \sin (\theta) X_{2}+\cos (\theta) Y_{2}, \text { and } \\
& Z=Z_{2}+\gamma .
\end{aligned}
$$

The third transformation entails a translation along the $X_{2}$ axis (denoted by the constant $\alpha$ ) which models the $X$ translation as defined by the parameter $x_{0}$ of Figure E-1 in Appendix $E$. The transformed coordinates are then defined as follows:

$$
\begin{gathered}
X_{3}=X_{2}-\alpha, \\
Y_{3}=Y_{2}, \text { and } \\
Z_{3}=Z_{2} .
\end{gathered}
$$

Figure F-3 depicts the situation modeled by equations (16)-(18).

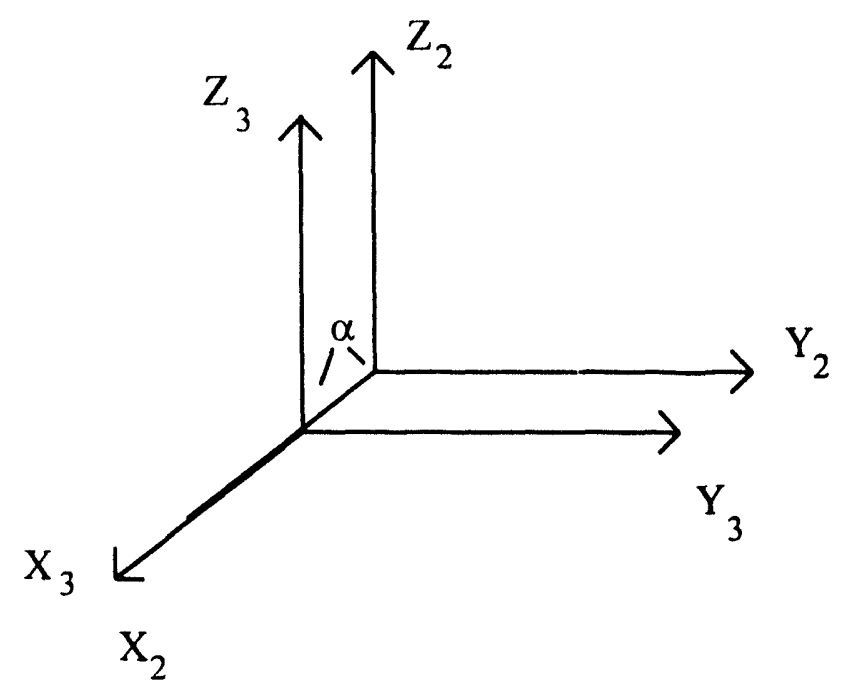

Figure F-3. Third Transformation - Translation Along $\mathbf{X}_{2}$ 
Inverting equations (16)-(18) and substituting into (13)-(15) provided a description of the original coordinates in terms of the three transformations:

$$
\begin{gathered}
X=\cos (\theta) X_{3}-\sin (\theta) Y_{3}+\cos (\theta) \alpha, \\
Y=\sin (\theta) X_{3}+\cos (\theta) Y_{3}+\sin (\theta) \alpha, \text { and } \\
Z=Z_{3}+\gamma .
\end{gathered}
$$

The fourth transformation is a translation along the $Y_{3}$ axis (denoted by the constant $\beta$ ) which models the $Y$ translation as defined by the parameter $y_{0}$ of Figure E-2 in Appendix E. The transformed coordinates are then defined as follows:

$$
\begin{gathered}
X_{4}=X_{3}, \\
Y_{4}=Y_{3}-\beta, \text { and } \\
Z_{4}=Z_{3} .
\end{gathered}
$$

Figure F-4 depicts the situation modeled by equations (22)-(24).

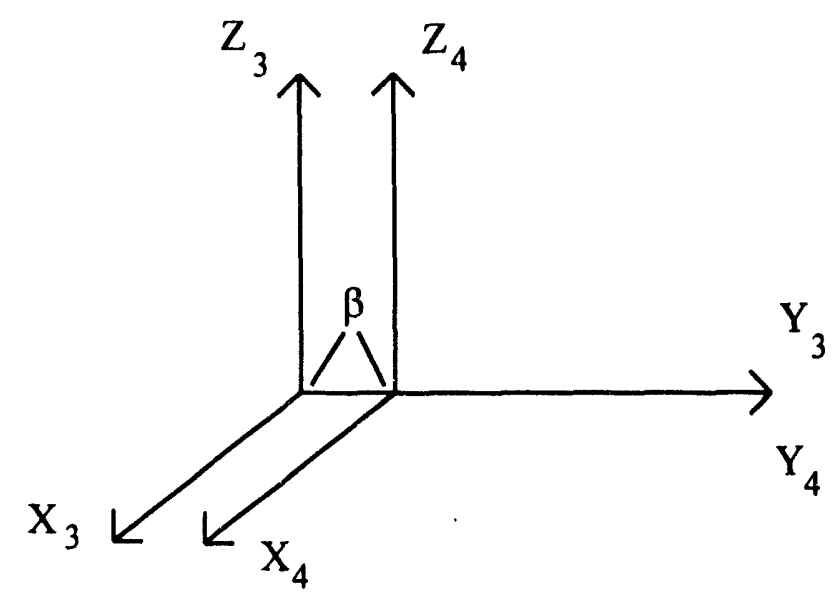

Figure F-4. Fourth Transformation - Translation Along $\mathbf{Y}_{\mathbf{3}}$

Inverting equations (22)-(24) and substituting into (19)-(21) provided a description of the original coordinates in terms of the four transformations:

$$
\begin{gathered}
X=\cos (\theta) X_{4}-\sin (\theta) Y_{4}+\cos (\theta) \alpha-\sin (\theta) \beta, \\
Y=\sin (\theta) X_{4}+\cos (\theta) Y_{4}+\sin (\theta) \alpha+\cos (\theta) \beta, \text { and } \\
Z=Z_{4}+\gamma .
\end{gathered}
$$

The fifth transformation is a rotation about the $\mathrm{Y}_{4}$ axis (denoted by the angle $\phi$ ) which models the tilt in the $\mathrm{X}$ direction as represented by $\theta_{X}$ in Figure E-1 of Appendix E. Figure F-5 depicts this situation as if the viewer is looking straight down the $\mathrm{Y}_{4}$ axis which is represented by the semi-shaded circle. 


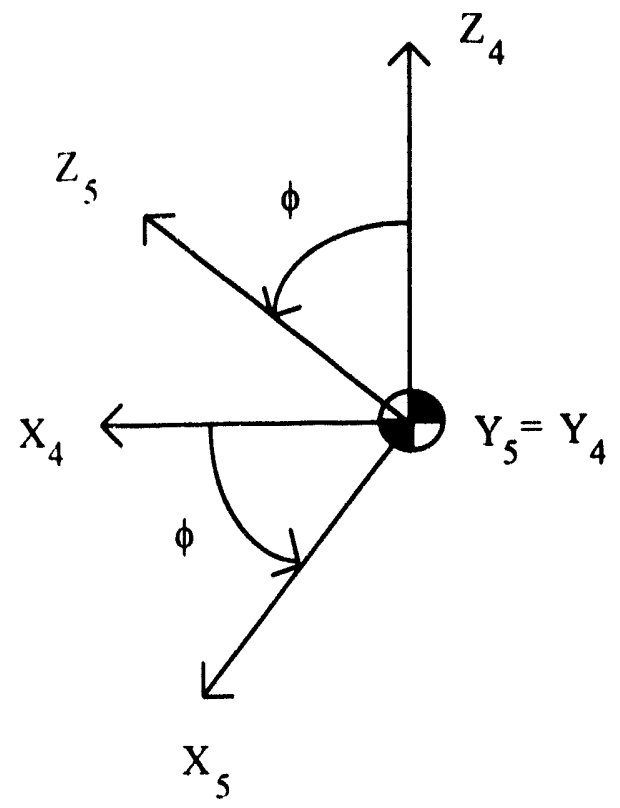

Figure F-5. Fifth Transformation - Rotation About $Y_{4}$

The axes $X_{5}, Y_{5}$, and $Z_{5}$ are therefore defined as follows:

$$
\begin{gathered}
X_{5}=\cos (\phi) X_{4}-\sin (\phi) Z_{4}, \\
Y_{5}=Y_{4} \text {, and } \\
Z_{5}=\sin (\phi) X_{4}+\cos (\phi) Z_{4} .
\end{gathered}
$$

Equations (31)-(33) result from inverting (29)-(30):

$$
\begin{gathered}
X_{4}=\cos (\phi) X_{5}+\sin (\phi) Z_{5}, \\
Y_{4}=Y_{5}, \text { and } \\
Z_{4}=-\sin (\phi) X_{5}+\cos (\phi) Z_{5} .
\end{gathered}
$$

Now/ substituting equations (31)-(33) into (25)-(27) allows a description of the original coordinate system:

$$
\begin{gathered}
X=\cos (\theta) \cos (\phi) X_{5}-\sin (\theta) Y_{5}+\cos (\theta) \sin (\phi) Z_{5}+\cos (\theta) \alpha-\sin (\theta) \beta, \\
Y=\sin (\theta) \cos (\phi) X_{5}+\cos (\theta) Y_{5}+\sin (\theta) \sin (\phi) Z_{5}+\sin (\theta) \alpha+\cos (\theta) \beta, \text { and } \\
Z=-\sin (\phi) X_{5}+\cos (\phi) Z_{5}+\gamma .
\end{gathered}
$$

The final transformation is a rotation about the $\mathrm{X}_{5}$ axis (denoted by the angle $\psi$ ) which models the tilt in the $Y$ direction as represented by $\theta_{y}$ in Figure E-2 of Appendix E. Figure F-6 depicts this situation as if the viewer is looking straight down the $X_{5}$ axis which is represented by the semi-shaded circle. 


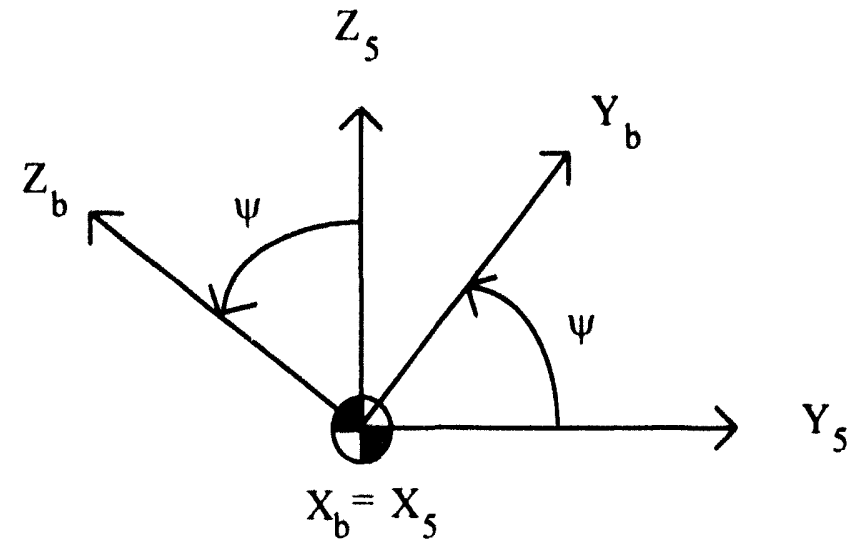

Figure F-6. Final Transformation - Rotation About $X_{5}$

The axes $X_{b}, Y_{b}$, and $Z_{b}$ are fixed in the body of interest and are defined as follows:

$$
\begin{gathered}
X_{b}=X_{5}, \\
Y_{b}=\cos (\psi) Y_{5}+\sin (\psi) Z_{5}, \text { and } \\
Z_{b}=-\sin (\psi) Y_{5}+\cos (\psi) Z_{5} .
\end{gathered}
$$

Equations (40)-(42) result from inverting (37)-(39):

$$
\begin{gathered}
X_{5}=X_{b}, \\
Y_{5}=\cos (\psi) Y_{b}-\sin (\psi) Z_{b}, \text { and } \\
Z_{5}=\sin (\psi) Y_{b}+\cos (\psi) Z_{b} .
\end{gathered}
$$

Now substituting equations (40)-(42) into (34)-(36) allows a transformation from the body-fixed coordinate system to the machine coordinates:

$$
\begin{gathered}
X=[\cos (\theta) \cos (\phi)] X_{b}+[\cos (\theta) \sin (\phi) \sin (\psi)-\sin (\theta) \cos (\psi)] Y_{b} \\
+[\cos (\theta) \sin (\phi) \cos (\psi)+\sin (\theta) \sin (\psi)] Z_{b}+\cos (\theta) \alpha-\sin (\theta) \beta, \\
Y=[\sin (\theta) \cos (\phi)] X_{b}+[\sin (\theta) \sin (\phi) \sin (\psi)+\cos (\theta) \cos (\psi)] Y_{b} \\
+[\sin (\theta) \sin (\phi) \cos (\psi)-\cos (\theta) \sin (\psi)] Z_{b}+\sin (\theta) \alpha+\cos (\theta) \beta, \text { and } \\
Z=-\sin (\phi) X_{b}+\cos (\phi) \sin (\psi) Y_{b}+\cos (\phi) \cos (\psi) Z_{b}+\gamma .
\end{gathered}
$$

Now if equations (43) and (44) are rewritten by collecting on functions of $\theta$, the following results:

$$
\begin{gathered}
X=\cos (\theta) r_{x}-\sin (\theta) r_{y}, \\
Y=\sin (\theta) r_{x}+\cos (\theta) r_{y},
\end{gathered}
$$




$$
\begin{gathered}
r_{x}=\cos (\phi) X_{b}+\sin (\phi) \sin (\psi) Y_{b}+\sin (\phi) \cos (\psi) Z_{b}+\alpha, \text { and } \\
r_{y}=\cos (\psi) Y_{b}-\sin (\psi) Z_{b}+\beta .
\end{gathered}
$$

\section{Small Angle and Offset Approximation}

Further analysis of equations (46)-(49) requires the simplifying assumptions of small angles and small offsets $(\phi, \psi, \alpha$, and $\beta)$. This small angle/offset approximation has been observed to be appropriate in the lab especially since these quantities are being driven to zero. All quadratic terms are neglected with this approximation and equations (48)-(49) provide the following approximations:

$$
\begin{aligned}
& r_{x} \cong X_{b}+\phi Z_{b}+\alpha, \text { and } \\
& r_{y} \cong Y_{b}-\psi Z_{b}+\beta
\end{aligned}
$$

It should be noted that $\theta$ and $\gamma$ are not small quantities and equations (46) and (47) are not changed. Now, equation (45) is solved for $Z_{b}$ and higher order terms neglected:

$$
Z_{b} \cong Z-\gamma+\phi X_{b}-\psi Y_{b} .
$$

Now, $Z_{b}$ needs to be eliminated from (50) and (51) and replaced with $Z$. This is required since $Z$ is the known quantity (height of the measurement points). Equation (52) is substituted into (50) and (51) and higher order terms are neglected:

$$
\begin{aligned}
& r_{x} \cong X_{b}+\phi(Z-\gamma)+\alpha=X_{b}+x_{s}, \text { and } \\
& r_{y} \cong Y_{b}-\psi(Z-\gamma)+\beta=Y_{b}+y_{s} .
\end{aligned}
$$

The quantities $x_{S}$ and $y_{S}$ were defined for notational ease and are small numbers.

\section{Converting to Polar Coordinates}

Now, at the lower measurement points, the laser displacement sensors are used to determine the offsets based on measurements in polar coordinates with the angle $\theta$ being the independent variable. Therefore, equations (46), (47), (53), and (54) must be converted to polar coordinates. The following relations hold for the table coordinate system:

$$
\begin{gathered}
X(\theta)=R(\theta) \cos (\theta), \text { and } \\
Y(\theta)=R(\theta) \sin (\theta) .
\end{gathered}
$$

And, for the body fixed coordinate system:

$$
\begin{gathered}
X_{b}\left(\theta_{b}\right)=R_{b}\left(\theta_{b}\right) \cos \left(\theta_{b}\right), \text { and } \\
Y_{b}\left(\theta_{b}\right)=R_{b}\left(\theta_{b}\right) \sin \left(\theta_{b}\right) .
\end{gathered}
$$


Equations (55) and (56) produce the following well known result and equations (46) and (47) further simplify the result upon substitution:

$$
R=\sqrt{X^{2}+Y^{2}}=\sqrt{r_{x}^{2}+r_{y}^{2}} .
$$

Substitution of (53) and (54) into (59) produces the following after neglecting higher order terms:

$$
R=\sqrt{\left(X_{b}+x_{s}\right)^{2}+\left(Y_{h}+y_{s}\right)^{2}}=\sqrt{\left(X_{b}^{2}+Y_{h}^{2}\right)+2\left(X_{b} x_{1}+Y_{h} y_{1}\right)} .
$$

Substitution of equations (57) and (58) into (60) produces the following:

$$
\begin{gathered}
R=\sqrt{R_{b}^{2}\left[\cos \left(\theta_{b}\right)^{2}+\sin \left(\theta_{b}\right)^{2}\right]+2 R_{b}\left[x_{s} \cos \left(\theta_{b}\right)+y_{s} \sin \left(\theta_{b}\right)\right]}= \\
R_{b} \sqrt{1+\frac{2}{R_{b}}\left[x_{s} \cos \left(\theta_{b}\right)+y_{s} \sin \left(\theta_{b}\right)\right]} .
\end{gathered}
$$

Now using the binomial theorem of expansion and neglecting higher order terms results in the following:

$$
R \cong R_{b}\left\{1+\frac{1}{R_{b}}\left[x_{s} \cos \left(\theta_{b}\right)+y_{s} \sin \left(\theta_{b}\right)\right]\right\}=R_{b}+x_{s} \cos \left(\theta_{b}\right)+y_{s} \sin \left(\theta_{b}\right) .
$$

The next step is to derive the relationship between $\theta$ and $\theta_{\mathrm{b}}$. Then the geometry of an idealized cone will be used to describe $R_{b}$.

\section{Derivation of Relationship Between $\theta$ and $\theta_{\mathbf{b}}$}

Since measurements are made in machine coordinates and the object geometry is described in body-fixed coordinates, a simplified relationship between the polar machine coordinate system and the polar bodyfixed coordinate system is required. This entails determining the relationship between the independent variables in the two coordinate systems (the measured angle in both systems $-\theta$ and $\theta_{b}$ ). A polar description of the offset between the two coordinate systems is needed to begin this derivation:

$$
\begin{gathered}
x_{0}=r_{0} \cos \left(\theta_{0}\right), \text { and } \\
y_{0}=r_{0} \sin \left(\theta_{0}\right) .
\end{gathered}
$$

Now $\mathrm{r}_{0}, \mathrm{x}_{0}$, and $\mathrm{y}_{0}$ are small quantities which are constant functions of $\phi, \psi, \alpha, \beta$, and the bias error $(\varepsilon)$. The specific form will be derived later and is included in equations (65) and (66) for completeness,

$$
\begin{aligned}
& x_{0}=\alpha+\phi(Z-\gamma-\varepsilon), \text { and } \\
& y_{0}=\beta-\psi(Z-\gamma-\varepsilon) .
\end{aligned}
$$

Based on a cross-section of the object, these quantities are related geometrically as shown in Figure F-7. 


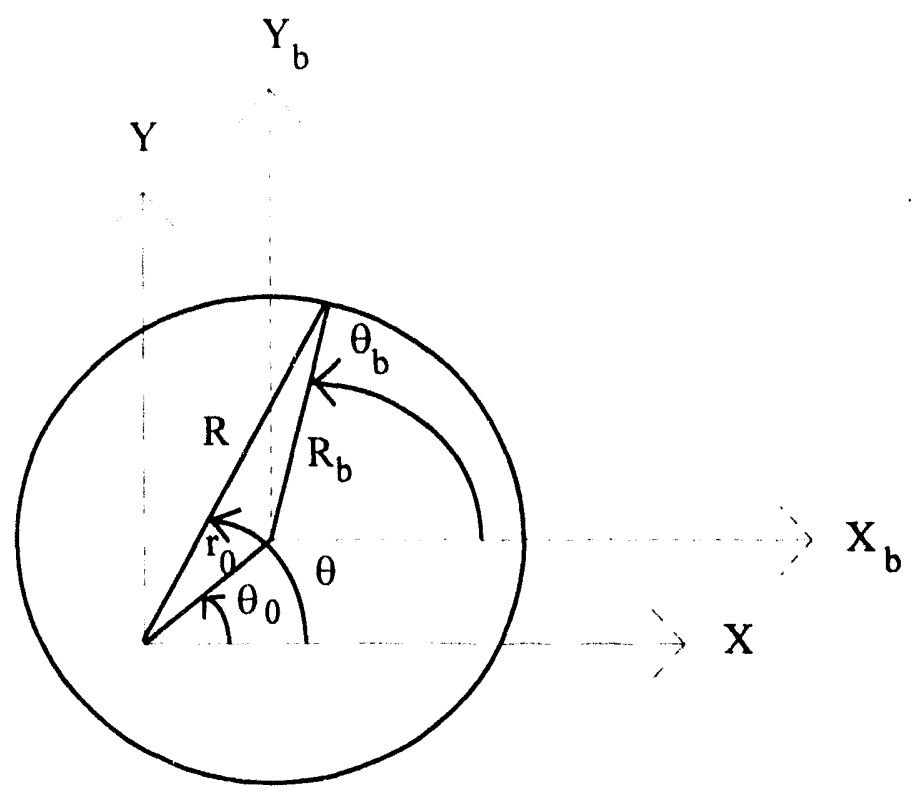

Figure F-7. Geometric Relationship Between $\theta$ and $\theta_{\mathbf{b}}$

Form Figure F-7 the following relationships are obvious:

$$
\begin{aligned}
& X=X_{b}+x_{0}, \text { and } \\
& Y=Y_{b}+y_{o} .
\end{aligned}
$$

Now the following relationship will be assumed (where the error term, $\theta_{\mathrm{e}}$, is to be determined):

$$
\theta_{b}=\theta+\theta_{e}
$$

Continuing the development:

$$
\tan \left(\theta_{b}\right)=\frac{Y_{b}}{X_{b}}=\frac{X-x_{0}}{Y-y_{0}}=\tan \left(\theta+\theta_{e}\right)=\frac{\tan (\theta)+\tan \left(\theta_{e}\right)}{1-\tan (\theta) \tan \left(\theta_{e}\right)} .
$$

And substituting for $\tan (\theta)$ :

$$
\frac{X-x_{0}}{Y-y_{0}}=\frac{\frac{Y}{X}+\tan \left(\theta_{e}\right)}{1-\frac{Y}{X} \tan \left(\theta_{e}\right)} .
$$

Now solving for $\tan \left(\theta_{e}\right)$ and neglecting higher order terms of $x_{0}$ and $y_{0}$ :

$$
\tan \left(\theta_{e}\right)=\frac{X\left(Y-y_{0}\right)-Y\left(X-x_{0}\right)}{X\left(X-x_{0}\right)+Y\left(Y-y_{0}\right)} \cong \frac{Y x_{0}-X y_{0}}{\left(X^{2}+Y^{2}\right)-\left(X x_{0}+Y y_{0}\right)}
$$


Substituting for $\mathrm{X}$ and $\mathrm{Y}$ and dividing by $\mathrm{R}^{2}$ results in the following:

$$
\tan \left(\theta_{e}\right)=\frac{R\left[x_{0} \sin (\theta)-y_{0} \cos (\theta)\right]}{R^{2}-R\left[x_{0} \cos (\theta)+y_{0} \sin (\theta)\right]}=\frac{\left(\frac{x_{0}}{R}\right) \sin (\theta)-\left(\frac{y_{0}}{R}\right) \cos (\theta)}{1-\left[\left(\frac{x_{0}}{R}\right) \cos (\theta)+\left(\frac{y_{0}}{R}\right) \sin (\theta)\right]}
$$

Now using the binomial theory of expansion and neglecting higher order terms the following results:

$$
\begin{gathered}
\tan \left(\theta_{e}\right) \cong\left\{1+\left[\left(\frac{x_{0}}{R}\right) \cos (\theta)+\left(\frac{y_{0}}{R}\right) \sin (\theta)\right]\right\}\left\{\left(\frac{x_{0}}{R}\right) \sin (\theta)-\left(\frac{y_{0}}{R}\right) \cos (\theta)\right\} \cong \\
\left(\frac{x_{0}}{R}\right) \sin (\theta)-\left(\frac{y_{0}}{R}\right) \cos (\theta) .
\end{gathered}
$$

Alternatively, (and recognizing that $\tan \left(\theta_{\mathrm{e}}\right)$ is a small number),

$$
\tan \left(\theta_{e}\right) \cong\left(\frac{r_{0}}{R}\right)\left[\cos \left(\theta_{0}\right) \sin (\theta)-\sin \left(\theta_{0}\right) \cos (\theta)\right]=\left(\frac{r_{0}}{R}\right) \sin \left(\theta-\theta_{0}\right) \cong \theta_{e}
$$

Hence the final result is as follows:

$$
\theta_{b}=\theta+\theta_{e} \cong \theta+\left(\frac{r_{0}}{R}\right) \sin \left(\theta-\theta_{0}\right) \cong \theta+\left(\frac{x_{0}}{R}\right) \sin (\theta)-\left(\frac{y_{0}}{R}\right) \cos (\theta) .
$$

Equation (76) is substituted into the sine and cosine functions and small angle approximations used:

$$
\begin{gathered}
\cos \left(\theta_{b}\right)=\cos \left(\theta+\theta_{e}\right)=\cos (\theta) \cos \left(\theta_{e}\right)-\sin (\theta) \sin \left(\theta_{e}\right) \cong \\
\cos (\theta)-\theta_{e} \sin (\theta)=\cos (\theta)-\frac{r_{0}}{R} \sin \left(\theta-\theta_{0}\right) \sin (\theta), \text { and } \\
\sin \left(\theta_{b}\right)=\sin \left(\theta+\theta_{e}\right)=\sin (\theta) \cos \left(\theta_{e}\right)+\cos (\theta) \sin \left(\theta_{e}\right) \cong \\
\sin (\theta)+\theta_{e} \cos (\theta)=\sin (\theta)+\frac{r_{0}}{R} \sin \left(\theta-\theta_{0}\right) \cos (\theta)
\end{gathered}
$$

Substituting equations (77) and (78) into (62) and neglecting higher order terms produces the following:

$$
R \cong R_{b}+x_{s} \cos (\theta)+y_{s} \sin (\theta) .
$$

\section{Idealized Geometry of a Test Part}

The geometry of the test part will be modeled as a right nearly-circular cone. Therefore the following relationship holds:

$$
R_{b}(\theta)=R_{\text {base }}(\theta)\left(1-\frac{Z_{b}}{h}\right)
$$


where $R_{b a s e}$ is the radius of the base as defined in Appendix D,

$h$ is the height of the cone, and

$Z_{b}$ is the height measurement.

Now $Z_{b}$ is replaced in equation (80) using (52)

$$
R_{b}(\theta)=R_{b a s e}(\theta)\left[1-\frac{(Z-\gamma)+\left(\phi X_{b}-\psi Y_{b}\right)}{h}\right]
$$

Now equations (57) and (58) are substituted into (81):

$$
R_{b}(\theta)=R_{\text {base }}(\theta)\left[1-\frac{(Z-\gamma)}{h}\right]-R_{\text {base }}(\theta)\left\{\frac{R_{b}\left[\phi \cos \left(\theta_{\mathrm{b}}\right)-\psi \sin \left(\theta_{\mathrm{b}}\right)\right]}{h}\right\} .
$$

Again substituting (81) into (82) and neglecting higher order terms provides the following:

$$
R_{b}(\theta)=R_{\text {base }}(\theta)\left[1-\frac{(Z-\gamma)}{h}\right]-\frac{R_{\text {base }}^{2}(\theta)}{h}\left[1-\frac{(Z-\gamma)}{h}\right]\left[\phi \cos \left(\theta_{\mathrm{b}}\right)-\psi \sin \left(\theta_{\mathrm{b}}\right)\right] \text {. }
$$

And finally, substituting (77) and (78) into (83) and neglecting higher order terms results in the following:

$$
\begin{gathered}
R_{b}(\theta)=R_{\text {base }}(\theta)\left[1-\frac{(Z-\gamma)}{h}\right]-\varepsilon[\phi \cos (\theta)-\psi \sin (\theta)], \text { and } \\
\varepsilon=\frac{R_{\text {base }}{ }^{2}(\theta)}{h}\left[1-\frac{(Z-\gamma)}{h}\right] .
\end{gathered}
$$

Now $\varepsilon$ is the bias error term due to the conical shape. It is instructive to produce an approximate value for $\varepsilon$ which is a constant. The best estimate of $R_{\text {base }}$ is the average radius at the base which can be measured approximately (denote as $\mathrm{R}_{0}$ ). Hence, substitute (84) and (85) into (79):

$$
R \cong R_{\text {base }}(\theta)\left[1-\frac{(Z-\gamma)}{h}\right]+\left[\left(x_{s}-\phi \varepsilon\right) \cos (\theta)+\left(y_{s}+\psi \varepsilon\right) \sin (\theta)\right]
$$

And substituting definitions for $x_{S}$ and $y_{s}$ into (86) and produce the final result:

$$
\begin{gathered}
R \cong R_{\text {base }}(\theta)\left[1-\frac{(Z-\gamma)}{h}\right]+[\phi(Z-\gamma-\varepsilon)+\alpha] \cos (\theta)+[-\psi(Z-\gamma-\varepsilon)+\beta] \sin (\theta), \\
\varepsilon=\frac{R_{0}^{2}}{h}\left[1-\frac{(Z-\gamma)}{h}\right] .
\end{gathered}
$$




\section{General Observations}

Some observations about the final form should be noted. The first term is the measure radius of the object at a given height, $Z$. The coefficients of the transcendental terms model the offsets due to misalignment. If a measurement is made at the tip of the cone then $\varepsilon$ is zero and no bias error is introduced. Also, if the object is cylindrical, the parameter $h$ is effectively infinity and again $\varepsilon$ is zero. The bias error effectively decreases the height to be used in interpreting the tilt angles. Also, the bias terms are dependent on the tilt angles and independent of the translation parameters, as is expected.

The offset terms can be related directly to the adjustment parameters discussed in Appendix E. The reference line angle for the $\mathrm{x}$ direction as defined in Figure $\mathrm{E}-1$ has the following description:

$$
\theta_{x}=\phi
$$

The reference line angle for the $y$ direction as defined in Figure E-2 has the following description:

$$
\theta_{y}=-\psi
$$

It should be recalled that $\theta_{y}$ from Figure E-2 was defined in a left-handed coordinate system which explains the minus sign. The $x$ direction intercept, $x_{0}$, of the reference line as defined in Figure $E-1$ has the following definition (to avoid confusion, this parameter will be denoted as $i_{X}$ in this appendix):

$$
x_{0}=\alpha-\phi \gamma=i_{x}
$$

The $y$ direction intercept of the reference line, $\mathrm{y}_{0}$, as defined in Figure $\mathrm{E}-2$ has the following definition (to avoid confusion, this parameter will be denoted as $i_{y}$ in this appendix):

$$
y_{0}=\beta+\psi \gamma=i_{y}
$$

The final section of this appendix will derive an alternate adjustment procedure which takes into account these bias errors.

\section{Calculation of Adjustment Parameters}

The adjustment parameters are calculated using measurements of reference axis offsets at two heights on the body. The $X$ and $Y$ offsets at the upper height will be denoted as $X_{u}$ and $Y_{u}$. The upper measurement height will be noted as $Z_{\mathrm{u}}$. The upper height measurement is made directly on the reference axis, therefore the bias error term for the upper measurement, $\varepsilon_{\mathrm{u}}$, is zero. The $\mathrm{X}$ and $\mathrm{Y}$ offsets at the lower height will be denoted as $X_{1}$ and $Y_{1}$. The lower measurement height will be noted as $Z_{1}$. The lower bias error term, $\varepsilon_{\mid}$, is non-zero and must be taken account of. Therefore, a modified lower height will be defined which takes this error term into account:

$$
Z_{\varepsilon}=Z_{l}-\varepsilon
$$

Now, using the information from the previous paragrapin as well as equations (87) (or (65) and (66)), and (89)-(93), the following equations can be defined:

$$
\begin{aligned}
& X_{u}=\hat{\theta}_{x} \bar{L}_{u}+i_{x}, \\
& X_{l}=\theta_{x} Z_{\varepsilon}+i_{x},
\end{aligned}
$$




$$
\begin{aligned}
& Y_{u}=\theta_{y} Z_{u}+i_{y}, \text { and } \\
& Y_{l}=\theta_{y} Z_{\varepsilon}+i_{y} .
\end{aligned}
$$

And these equations can be solved for the adjustment parameters:

$$
\begin{gathered}
\theta_{x}=\frac{X_{u}-X_{l}}{Z_{u}-Z_{\varepsilon}}, \\
i_{x}=\frac{X_{l} Z_{u}-X_{u} Z_{\varepsilon}}{Z_{u}-Z_{\varepsilon}}, \\
\theta_{y}=\frac{Y_{u}-Y_{l}}{Z_{u}-Z_{\varepsilon}}, \text { and } \\
i_{y}=\frac{Y_{l} Z_{u}-Y_{u} Z_{\varepsilon}}{Z_{u}-Z_{\varepsilon}} .
\end{gathered}
$$

The adjustment parameters are calculated using equations (97)-(101). If the bias error term is not used then the denominators will be smaller and the calculated adjustment parameters would generally be larger. Hence, the bias error term tends to decrease the adjustments needed. This observation is supported by laboratory experience. Typically, adjustments for a given iteration will in the opposite direction of the adjustments required for the previous iteration. 


\section{REFERENCES}

1. R. Boynton, R. Bell, and K. Wiener, "Using Helium to Predict the Mass Properties of an Object in the Vacuum of Space," Proceedings of the 50th Annual Conference of the Society of Allied Weight Engineers, Inc., San Diego, CA, May 20-23, 1991.

2. "Model POI-50Z Operating Instructions," Space Electronics, Inc., Berlin, CT, 1991.

3. D. F. Riddle, Calculus and Analytic Geometry, 3rd edition, Wadsworth, Belmont, CA, 1979.

4. "Expertvision User's Manual," Motion Analysis Corporation, Santa Rosa, CA, 1990.

5. "ADA-16 Analog Data Acquisition System Operating Manual," Motion Analysis Corporation, Santa Rosa, CA, 1990.

6. "386-MATLAB User's Guide," The Math Works, Inc., Natick, MA, 1991.

7. "VP110 Video Processor Operator's Manual," Motion Analysis Corporation, Santa Rosa, CA, 1990.

8. "MQ Laser Analog Sensor Instruction Manual," Matsushita Electric Works, Ltd., Kadoma-shi, Osaka, Japan, 1986.

9. W. A. Wilson and J. I. Tracy, Analytic Geometry, 3rd edition, Heath, Boston, MA. 


\section{DISTRIBUTION:}

1 Richard Boynton

Space Electronics, Inc.

81 Fuller Way

Berlin, Connecticut 06037

2 Mrs. Elaine Alspach

U.S. Army Space and Strategic Defense Cmd.

P.O. Box 1500, ATTN: CSSD-TE-P

Huntsville, Alabama 35807-3801

3 Mr. Randall W. Carpenter

U.S. Army Space and Strategic Defense Cmd.

P.O. Box 1500, ATTN: CSSD-TE-S

Huntsville, Alabama 35807-3801

4 Major Nat Thongchua

BMDO/GST

The Pentagon

Room E180

Washington, DC 20301-7100

5. SMC/CUC

Att: Major Robert L. Kelsey

160 Skynet St.

Suite 1536A

Los Angeles AFB, CA $90245-4683$

12700

2707

2741

2741

2742

2742

8283

9800

9811

9811

9811

9811

9811

9812

9812

9813

9818

9818

8523-2

7141

7151

$10 \quad 7613-2$
R. A. David

V. Gabbard

T. J. Baca

G. H. James

J. R. Garcia

R. C. Varga

D. P. Green

D. J. Rigali

J. L. McDowell

R. W. Escapule

J. J. Stanalonis

J. E. Suazo

D. M. VanZuiden

D. L. Keese

M. S. Krein

G. J. Hochrein

A. K. Miller

R. D. Lohr

Central Technical files

Technical Library

Technical Publications

Document Processing for DOE/OSTI 

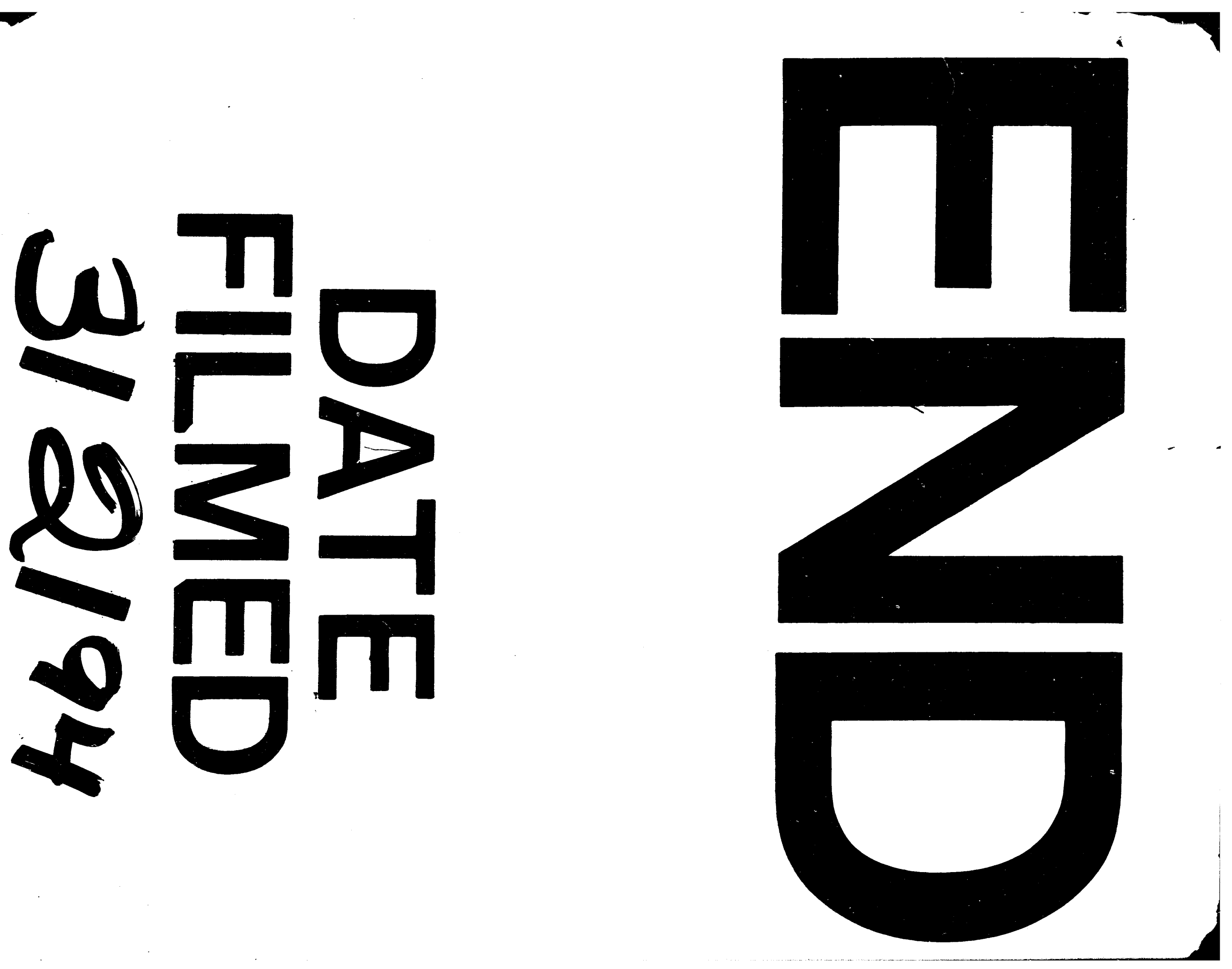
